\title{
Identification, Validation, and Functional Annotations of Genome-Wide Profile Variation between Melanocytic Nevus and Malignant Melanoma
}

\author{
Wei Han $\mathbb{D}^{1,2}$ Wen-Hao Xu, ${ }^{3,4}$ Jian-Xiong Wang, ${ }^{1,2}$ Jia-Min Hou, ${ }^{1,2}$ Hai-Liang Zhang $\mathbb{D},{ }^{3,4}$ \\ Xiao-Yu Zhao $\mathbb{D}^{1,2}$ and Guo-Liang Shen ${ }^{1,2}$ \\ ${ }^{1}$ Department of Burn and Plastic Surgery, The First Affiliated Hospital of Soochow University, Suzhou 215000, China \\ ${ }^{2}$ Department of Surgery, Soochow University, Suzhou 215000, China \\ ${ }^{3}$ Department of Urology, Fudan University Shanghai Cancer Center, Shanghai 200032, China \\ ${ }^{4}$ Department of Oncology, Shanghai Medical College, Fudan University, Shanghai 200032, China
}

Correspondence should be addressed to Hai-Liang Zhang; zhangh1918@163.com, Xiao-Yu Zhao; 13776125762@163.com, and Guo-Liang Shen; sdfyysgl@163.com

Wei Han, Wen-Hao Xu, and Jian-Xiong Wang contributed equally to this work.

Received 13 February 2020; Revised 10 July 2020; Accepted 21 July 2020; Published 31 August 2020

Academic Editor: Kazim Husain

Copyright $\odot 2020$ Wei Han et al. This is an open access article distributed under the Creative Commons Attribution License, which permits unrestricted use, distribution, and reproduction in any medium, provided the original work is properly cited.

Cutaneous melanoma (CM) is known as an aggressive malignant cancer; some of which are directly derived from melanocytic nevi, which have been attracting growing attention from the last decades. This study focused on comprehensive identification, validation, and functional annotations of prognostic differentially expressed genes (DEGs) between melanocytic nevus and malignant melanoma in genome-wide profiles. DEGs were obtained using three chip datasets from GEO database to identify after standardization annotation. A total of 73 DEGs were identified as possible candidate prognostic biomarkers between melanocytic nevus and malignant melanoma. In addition, survival curves indicated that six hub genes, including $F A B P 5, I V L$, KRT6A, KRT15, KRT16, and TIMP2, were significant prognostic signatures for CM and of significant value to predict transformation from nevi to melanoma. Furthermore, immunohistochemistry staining was performed to validate differential expression levels and prognostic implications of six hub genes between CM tissue and nevus tissues from the First Affiliated Hospital of Soochow University cohort. It suggested that significantly elevated FABP5, IVL, KRT6A, KRT15, KRT16, and TIMP2 proteins expressed in the CM than in the nevus tissues. Functional enrichment and significant pathways of the six significant hub genes indicated that the mostly involved hallmarks include the P53 pathway, K-ras signaling, estrogen response late, and estrogen response early. In summary, this study identified significant DEGs participating in the process of malignant transformation from nevus to melanoma tissues based on comprehensive genomic profiles. Transcription profiles of FABP5, IVL, KRT6A, KRT15, KRT16, and TIMP2 provided clues of prognostic implications, which might help us evaluate malignant potential of nevus and underlying carcinogenesis progress from melanocytic nevus to melanoma.

\section{Introduction}

Cutaneous melanoma (CM) is an aggressive tumor that is the fifth and sixth most common malignant tumor of men and women, respectively [1]. Worldwide, cutaneous melanoma accounts for 55,500 cancer deaths ( $0.7 \%$ mortality rate) every year [2]. Further, an elevated incidence and aggressive prognosis of melanoma are associated with the metastatic phase, accounting for the 15\% 5-year survival rate [3]. The preferred treatment regimen for melanoma is surgical resection of the primary tumor, while metastatic melanoma is much more difficult to treat with radiotherapy and chemotherapy [4]. 
Recently developed immunotherapies and targeted therapies show promise for improving the prognosis of patients with advanced melanoma [5]. Identification of melanomaassociated oncogenes informs different therapeutic strategies, and small molecule inhibitors are available to target specific proteins involved in the pathogenesis of melanoma [3]. For example, the outcome of a phase II randomized clinical trial suggests that the BRAF inhibitor vemurafenib prolongs the survival of patients with advanced melanoma carrying the $B R A F^{V 600 E}$ mutation [6].

Melanocytic nevus, the most common benign skin tumor of humans, is caused by an increase in melanocyte populations in the epidermis and dermis [7]. The growth of melanocyte nevus is regulated by genetic profiles. For example, evidence indicates that clonal melanocyte tumors are triggered by specific oncogenic mutations in components of the mitogenactivated protein kinase (MAPK) signal transduction pathway, most commonly the $B R A F^{V 600 E}$ that constitutively activates the protein kinase activity of BRAF $[8,9]$. According to the Clark model, the pathogenesis of melanoma assumes that numerous steps are required for the progression from melanocytes to malignant melanoma [10], including formation of banal nevi, dysplastic nevi, melanoma in situ, and invasive melanoma.

Approximately $25 \%$ of cutaneous melanomas arise from nevi [11], which may approach $50 \%$ in potentially high-risk patients with numerous nevi [12]. Also, ultraviolet radiation will increase melanoma risk, especially among persons with a high nevus count [9]. In melanomas arise from preexisting nevi, the residual original nevi are usually histologically apparent [7]. The results of genetic analysis of benign nevusmelanoma pairs that are consistent with histological findings support the hypothesis that melanoma cells are directly derived from nevus cells $[9,13]$. Thus, there is an urgent need to understand the molecular mechanisms involved in the pathogenesis, progression, and recurrence of melanomas. Such efforts are enhanced through the identification of genes that are differentially expressed (DEGs) in melanocytic nevus vs. malignant melanoma. This information will help guide the development of precise diagnostic and therapeutic strategies.

As a rapidly emerging discipline, bioinformatics studies the collection, processing, storage, dissemination, analysis, and interpretation of biological information, to integrate life sciences and computer sciences. Bioinformatics mainly focuses on genomics and proteomics to identify genotypes and phenotypes associated with immune infiltration, tumorigenesis, and progression of melanoma to guide the development of targeted therapy [14]. Here, we analyzed three mRNA microarray datasets from the Gene Expression Omnibus (GEO) data repository to distinguish between melanocytic nevi and melanomas. Subsequently, we performed functional pathway enrichment analysis to further identify the mechanisms underlying malignant transformation. Protein-protein interaction (PPI) network analysis revealed the specific functions of the proteins to evaluate the importance of their interactions potentially associated with the malignant phenotype [15-17]. Furthermore, immunohistochemistry staining was performed to validate differential expression levels and prognostic implications of six hub genes between CM tissue and nevus tissues from the First Affiliated Hospital of Soochow University cohort.

Here, we focused on determining gene expression profiles to identify candidate diagnostic and prognostic biomarkers that may improve the treatment of patients with melanoma as well as illuminate the underlying biological interaction networks. Our findings led us to hypothesize that six significant hub genes, which may contribute to oncogenic activity, are significantly associated with poor prognosis of melanoma. These findings will facilitate efforts to develop new prognostic markers and therapeutic targets that distinguish high-risk nevi.

\section{Materials and Methods}

2.1. Patients and Variables. A total of 31 nevus and $31 \mathrm{CM}$ tissues were obtained from 62 patients at the Department of Burn and Plastic Surgery, the First Affiliated Hospital of Soochow University (FAHSU, Suzhou, China) from March 2016 to August 2019. None of the patients had received radiotherapy or chemotherapy before operation. Tissue samples, including nevus and melanoma tissue, were collected during surgery and fixed in $4 \%$ paraformaldehyde, available from FAHSU tissue bank. Clinical data was available to obtain from hospital records. This research was supported by the Independent Ethics Committee (IEC) of the FAHSU, and all patients were well informed of storing and upcoming use of their resected specimens for further research purposes.

\subsection{Acquisition and Standardization of Raw Microarray} Dataset. mRNA microarray datasets were screened and obtained from GEO (http://www.ncbi.nlm.nih.gov/geo) [18] testing mRNA expression in melanoma and nevus patients including three chip datasets GSE3189 [19], GSE12391 [20], and GSE46517 [21] (18 nevus and 45 melanoma samples in GSE3189, 18 nevus and 23 melanoma samples in GSE12391, and 9 nevus and 31 melanoma samples in GSE46517) in Affymetrix GPL96 platform. Significant DEGs distinguishing between melanoma and nevus tissues were identified using the limma $\mathrm{R}$ package across background correction of probe annotations. Adjusted $p$ values (adj. $p$ ), false discovery rate (FDR), and fold change were used for filtering of DEGs and applying to balancing statistically algorithm. $\mid \log _{2} \mathrm{FC}$ (fold change) $\mid>1.00$ and adj. $p$ value $<0.01$ were considered of statistical significance.

2.3. Functional Enrichment of DEGs. The Database for Annotation, Visualization, and Integrated Discovery (DAVID, https://david.ncifcrf.gov/) was utilized to perform functional and pathway enrichment analysis. It can provide systematic and integrative functional annotation tools for users to unravel biological meaning behind the list of genes. Gene ontology (GO) analysis including the biological process (BP), cellular component (CC), and molecular function (MF) and Kyoto Encyclopedia of Genes and Genomes (KEGG) pathway enrichment analysis were conducted for the selected DEGs by DAVID $[22,23]$. $p$ value $<0.05$ was considered statistically significant. 
2.4. PPI Network Construction and Analysis. In this study, STRING (http://string-db.org; version 11.0) was used to describe protein coregulation of DEGs and measure functional interactions among nodes [24]. The interaction specificity score $>0.4$ (the default threshold in the STRING database) was considered statistically significant.

Cytoscape (version 3.5) was utilized to visualize interaction networks obtained from STRING [25]. MCODE (version 1.4.2) of Cytoscape is a plug-in to cluster a given network to identify densely connected regions based on topology [26]. It was utilized to find the most related module network with selection threshold as follows: MCODE scores $>5$, degree cut-off $=2$, node score cut-off $=0.2$, Max depth $=100$, and $k$ score $=2$. The hub nodes of network with connectivity degrees $>10$ were selected. Network of 10 genes and neighbor nodes were obtained using cBioPortal (http://www .cbioportal.org/) tool [27]. GO: BP, CC, MF, and KEGG functional enrichment were analyzed and plotted using ClueGO (version 2.5.3) and CluePedia (version 1.5.3) [28].

2.5. TCGA Database. A total of $481 \mathrm{CM}$ patients with clinical profiles, among which $475 \mathrm{CM}$ patients with available RNA sequence data downloaded from UCXC (https://xenabrowser .net/datapages/) [29], were consecutively recruited in analyses. Phenotype and transcriptional expression profiles in 481 melanoma patients from TCGA were analyzed and displayed. Clinical and pathological parameters of the cohort were summarized. The 6 prognostic hub genes were identified as dichotomous variables with the median expression.

Gene Expression Profiling Interactive Analysis (GEPIA, http://gepia.cancer-pku.cn/) is a web tool that can provide fast and customizable functionalities based on data from The Cancer Genome Atlas (TCGA; https://tcga-data.nci.nih.gov/tcga/) and the Genotype-Tissue Expression project (GTEx; https:// www.gtexportal.org/home/index.html) [30]. GEPIA performs survival analysis based on gene expression levels, using logrank test for the hypothesis evaluation. The horizontal axis $(x$-axis) represented time in days, and the vertical axis $(y$ -axis) showed the probability of surviving or the proportion of people surviving. The dotted lines represented the $95 \%$ confidence interval information in the survival plots, with high expression marked in red and low expression marked in blue. The lines presented survival curves of two groups.

Multivariate analysis was applied with Cox regression models using BACK-LR methods to identify the variables, including Clark level (ref. I-III), pT stage (ref. T1-T2), pN stage (ref. N0), pM stage (ref. M0), pathological stage (ref. I-II), FABP5 expression (ref. Low), IVL expression (ref. Low), KRT6A expression (ref. Low), KRT15 expression (ref. Low), KRT16 expression (ref. Low), TIMP2 expression (ref. Low), and two known prognostic biomarkers of CM: S100B expression (ref. Low) and WNT5A expression (ref. Low). $p$ values less than 0.05 were considered significant in all tests.

2.6. Immunohistochemistry (IHC). Protein expression levels of significant six hub genes were measured using IHC staining and mouse monoclonal anti-FABP5 antibody (ab84028), antiinvolucrin antibody [SY5] (ab68), anti-cytokeratin 6 antibody [Ks6.KA12] (ab18586), anti-cytokeratin 15 antibody
[EPR1614Y] (ab52816), anti-cytokeratin 16/K16 antibody [EP1615Y] (ab76416), and anti-TIMP2 antibody (ab180630). Positive or negative staining of a certain protein in one FFPE slide was independently assessed by two experienced pathologists and supervised by a clinician. Based on the staining intensity level (no staining, weak, moderate, and strong staining), the score was ranging from 0 to 3 , as previously described [31]. The staining extent was graded from 0 to 4 for the coverage percentage of immunoreactive tumor cells $(0 \%, 1-25 \%$, $26-50 \%, 51-75 \%, 76-100 \%)$. The overall IHC score grading from 0 to 12 was evaluated according to the multiply of the staining intensity and extent score. Negative staining represented grade 0 to 4 and positive staining from 5 to 12 for each sample.

2.7. Transcription Factor Network and Data Processing of Gene Set Enrichment Analysis (GSEA). Transcription factor regulation networks were constructed in FABP5, IVL, KRT6A, KRT15, KRT16, and TIMP2 using R software (version 3.3.2). Significant nodes involved in coregulation of FABP5, IVL, KRT6A, KRT15, KRT16, and TIMP2 were described in circle plots (including transcription factor regulation-DNA binding, transcription factor regulation-activation, related lncRNA, targeted miRNA, and protein-protein interaction). Based on data from the TCGA database, GSEA tool (version 2.10.1 package) was used to predict associated up- and downregulated genes and their significantly involved hallmark pathways [32]. For each separate analysis, Student's $t$-test statistical score was performed in consistent pathways and the mean of the differential expression genes was calculated. A permutation test with 1000 times was used to identify the significantly changed pathways. The adj. $p$ using the Benjamini and Hochberg (BH) false discovery rate (FDR) method by default was applied to correct for the occurrence of false positive results. Significant related genes were defined with an adj. $p$ less than 0.01 and FDR less than 0.25 .

\section{Results}

3.1. Clinical and Pathologic Characteristics Baseline of CM Patients from TCGA and FAHSU. $481 \mathrm{CM}$ patients were enrolled from TCGA cohort and 31 from FAHSU cohort. Clinicopathological parameters of both nevus and CM patients from two cohorts, including age at surgery, gender, location, Clark level, Breslow's depth, TNM stage, and pathologic stage, are shown in Table 1.

3.2. Screening and Identification of DEGs between Melanoma and Nevus. DEGs (2,482 in GSE3189, 885 in GSE12391, and 1,218 in GSE46571) were selected after screening and standardization by using the limma package of R. Among the three datasets, 73 genes were overlapped in the Venn algorithm (Figure 1(a)) (Supplementary Table 1) between melanocytic nevus and malignant melanoma. The flowchart is presented in Figure 2.

3.3. Functional Enrichment Assessment. As shown in Supplementary Figure 1, GO analysis indicated that changes in biologic processes significantly enriched in ectoderm development, epidermis development, negative regulation of 
TABLE 1: Clinicopathological characteristics of the patients from two cohorts.

\begin{tabular}{|c|c|c|c|}
\hline \multirow[b]{2}{*}{ Characteristics } & \multicolumn{2}{|c|}{ FAHSU cohort } & \multirow[b]{2}{*}{$\begin{array}{l}\text { TCGA cohort } \\
(N=481)\end{array}$} \\
\hline & $\begin{array}{c}\text { Nevi } \\
(N=31)\end{array}$ & $\begin{array}{l}\text { Melanoma } \\
(N=31)\end{array}$ & \\
\hline \multicolumn{4}{|l|}{$N(\%)$} \\
\hline \multicolumn{4}{|l|}{ Age } \\
\hline$\leq 60$ years & $23(74.2)$ & $13(41.9)$ & $258(54.7)$ \\
\hline$>60$ years & $8(25.8)$ & $18(58.1)$ & $214(45.3)$ \\
\hline \multicolumn{4}{|l|}{ Gender } \\
\hline Male & $14(45.2)$ & $20(64.5)$ & 297 (61.9) \\
\hline Female & $17(54.8)$ & $11(35.5)$ & $183(38.1)$ \\
\hline \multicolumn{4}{|l|}{ Location } \\
\hline Extremity & $21(67.7)$ & $24(77.4)$ & - \\
\hline Trunk & $10(32.3)$ & $7(22.6)$ & - \\
\hline \multicolumn{4}{|l|}{ Clark level } \\
\hline I & - & $13(41.9)$ & $6(1.8)$ \\
\hline II & - & $15(48.4)$ & $18(5.5)$ \\
\hline III-IV & - & $3(9.7)$ & $246(75.5)$ \\
\hline $\mathrm{V}$ & - & $0(0)$ & $56(17.2)$ \\
\hline \multicolumn{4}{|c|}{ Breslow's depth (mm) } \\
\hline$\leq 0.75$ & - & $5(16.1)$ & $36(10.2)$ \\
\hline $0.76-1.50$ & - & $10(32.2)$ & $65(18.4)$ \\
\hline $1.51-4.00$ & - & $13(41.9)$ & $106(30.0)$ \\
\hline$>4.00$ & - & $3(9.8)$ & $146(41.4)$ \\
\hline \multicolumn{4}{|l|}{ pT stage } \\
\hline T1-T2 & - & $19(61.3)$ & $121(32.7)$ \\
\hline T3-T4 & - & $12(38.7)$ & $249(67.3)$ \\
\hline \multicolumn{4}{|l|}{$\mathrm{pN}$ stage } \\
\hline No & - & $31(100)$ & $236(65.0)$ \\
\hline N1 & - & $0(0)$ & $75(20.7)$ \\
\hline $\mathrm{N} 2$ & - & $0(0)$ & $52(14.3)$ \\
\hline \multicolumn{4}{|l|}{ pM stage } \\
\hline M0 & - & $31(100)$ & $424(94.4)$ \\
\hline M1 & - & $0(0)$ & $25(5.6)$ \\
\hline \multicolumn{4}{|l|}{ Pathologic stage } \\
\hline I-II & - & $31(100)$ & $233(53.9)$ \\
\hline III-IV & - & $0(0)$ & $199(46.1)$ \\
\hline \multicolumn{4}{|c|}{ Persistent distant metastasis } \\
\hline No & - & $31(100)$ & $217(46.1)$ \\
\hline Yes & - & $0(0)$ & $254(53.9)$ \\
\hline \multicolumn{4}{|c|}{ Cell atypia/atypical hyperplasia } \\
\hline No & $31(100)$ & - & - \\
\hline Yes & $0(0)$ & - & - \\
\hline
\end{tabular}

FAHSU: the First Affiliated Hospital of Soochow University; TCGA: The Cancer Genome Atlas.

signal transduction, and negative regulation of cell communication. Changes in cellular components were mainly enriched in the non-membrane-bounded organelle and intracellular non-membrane-bounded organelle. Function annotations were mostly enriched in structural molecule activity and structural constituent of cytoskeleton.
3.4. PPI Network Establishment. We constructed the coregulated network of DEGs (Figure 1(b)) and subsequently found the most significant module panel by using plug-in MCODE of Cytoscape (Figure 1(c)). With DAVID functional analysis of 10 hub genes, enrichment profiles suggested that hub genes in this module were primarily enriched in ectoderm development, epidermis development, structural molecule activity, structural constituent of cytoskeleton, and cytoskeleton (Table 2).

3.5. Hub Gene Selection and Analysis. After statistical selection, SPRP1A, S100A8, S100A9, CTSB, FABP5, IVL, KRT6A, KRT15, KRT16, and TIMP2 were verified as hub genes. Transcriptional coregulation of the ten hub genes and their neighbor nodes was set up visually (Figure 3(a)). GO: BP and KEGG functional annotation is displayed in Figure 3(b), with detailed function annotations listed in pie charts (Supplementary Figure 2). 55.56\% terms belonged to cornification, $33.33 \%$ to vesicle lumen, and $11.11 \%$ to intermediate filament cytoskeleton. Heat map, based on TCGA cohort $(n=475)$, showed that potential coexpression relationship may be found in the 6 significant hub genes, which could suggest basic value in prognostic prediction (Figure 3(c)).

3.6. Clinicopathological Statistical Analysis and Survival Outcomes in CM Patients from TCGA. Significant survival outcomes (OS: $p<0.05$ ) were found in Figures 4(a)-4(f). FABP5, IVL, KRT6A, KRT15, KRT16, and TIMP2 expression profiles suggested relatively significant elevated expression in tumor tissues compared with the corresponding normal tissues. In addition, multivariate Cox regression analysis of OS and PFS in TCGA cohort using BACK-LR methods was performed (Supplementary Table 2). In the COX models, we recruited two known prognostic biomarkers of melanoma, S100B, and WNT5A, to better identify the prognostic value of the hub genes. As shown in OS analysis, KRT6A along with S100B and WNT5A was considered statistically significant. FABP5 and S100B showed significant prognostic value in PFS analysis.

Except FABP5, elevated expression patterns of IVL, KRT6A, KRT15, KRT16, and TIMP2 were significantly associated with $\mathrm{T}$ stage (T1-T2 vs. T3-T4). In addition, six prognostic hub genes are highly expressed in primary than metastatic sites, plotted in Figure 5.

3.7. IHC Staining Analyses in 62 Patients from FAHSU Cohort. Next, to validate differential expressions of six prognostic hub genes between CM tissues and nevus tissues, we performed IHC analysis and found significantly elevated FABP5, IVL, KRT6A, KRT15, KRT16, and TIMP2 protein expressions in the CM than in the nevus tissues. The results and the scatter plots of IHC score are illustrated in Figure 6.

3.8. Significant Genes and Pathway Obtained by GSEA. Transcriptional regulation networks among FABP5, IVL, KRT6A, KRT15, KRT16, and TIMP2 are displayed in Figure 7. Significantly involved nodes (including transcription factor regulation-DNA binding, transcription factor regulation-activation, related lncRNA, targeted miRNA, and protein-protein interaction) were marked in different colors. Subsequently, a 


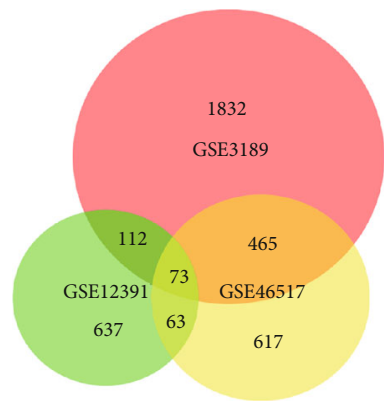

(a)

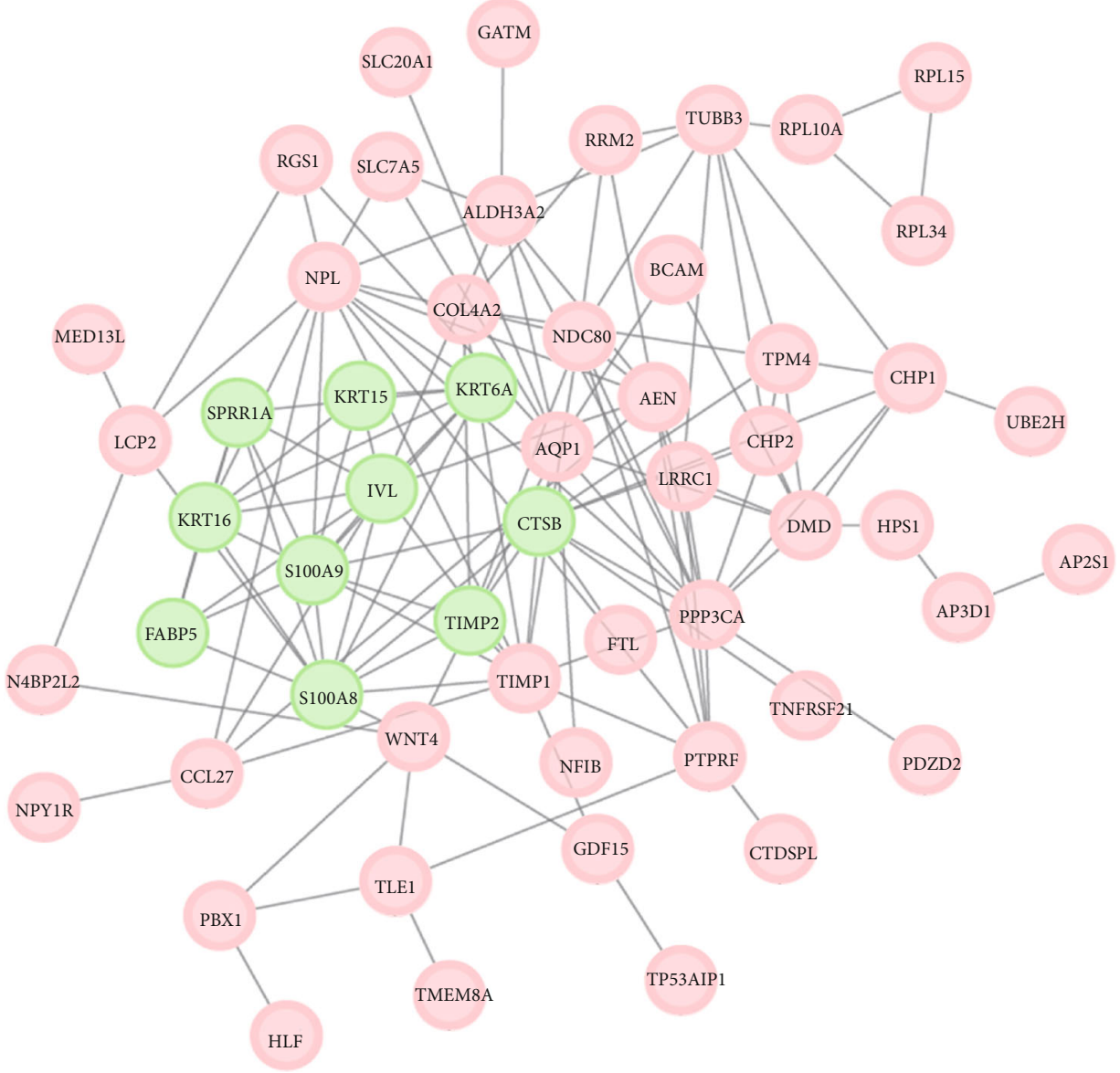

(b)

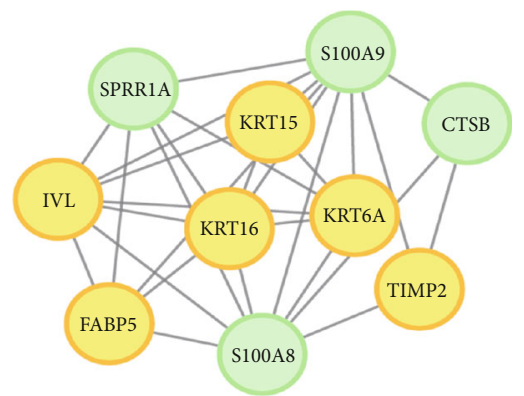

(c)

FIGURE 1: Venn diagram, PPI network, and the most significant module of DEGs. DEGs were selected with $\mid \log _{2}$ FC $\mid>1$ and adj. $p$ value $<0.01$ among the mRNA expression profiling chip datasets GSE3189, GSE12391, and GSE46517. (a) The three datasets showed an overlap of 73 genes in Venn diagram. (b) The PPI network of DEGs was constructed by using Cytoscape. (c) The most significant module was obtained from PPI network with 10 nodes. 


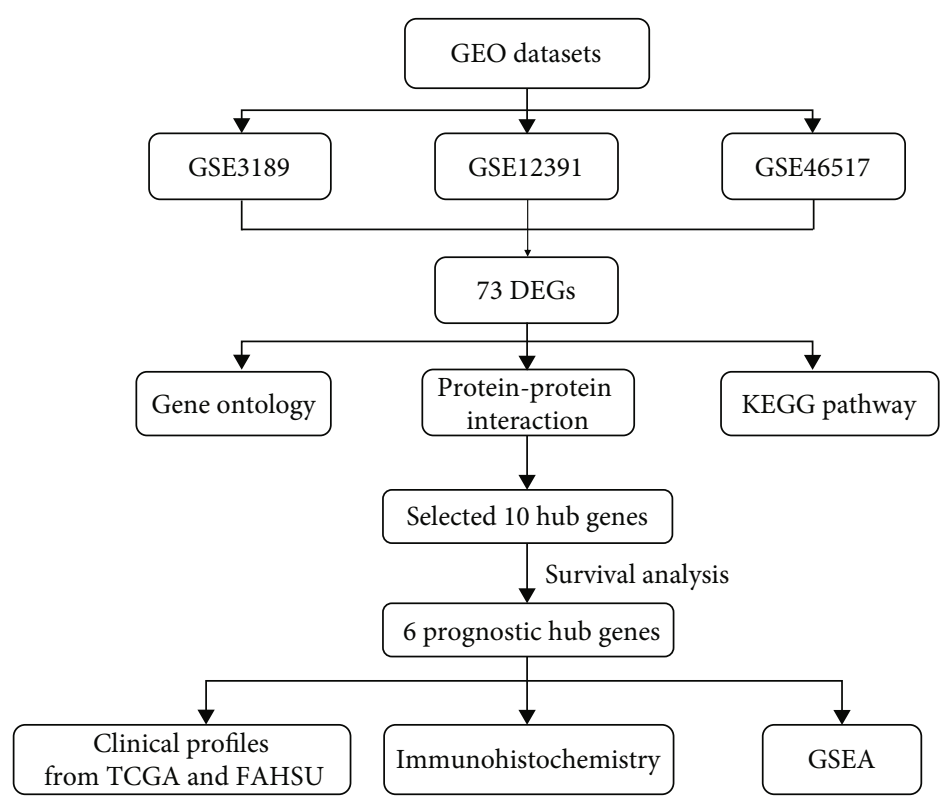

FIGURE 2: Flowchart of bioinformatics analysis. DEGs: differentially expressed genes; KEGG: Kyoto Encyclopedia of Genes and Genomes; GSEA: gene set enrichment analysis.

TABLE 2: GO enrichment analysis of DEGs in the most significant module.

\begin{tabular}{|c|c|c|c|}
\hline Term & Description & Count in gene set & $p$ value \\
\hline GO:0007398 & Ectoderm development & 6 & $7.87 E-08$ \\
\hline GO:0008544 & Epidermis development & 5 & $3.96 E-06$ \\
\hline GO:0005198 & Structural molecule activity & 5 & $5.83 E-04$ \\
\hline GO:0005200 & Structural constituent of cytoskeleton & 3 & $1.12 E-03$ \\
\hline GO:0005856 & Cytoskeleton & 5 & $3.62 E-03$ \\
\hline GO:0005882 & Intermediate filament & 3 & $4.08 E-03$ \\
\hline GO:0045111 & Intermediate filament cytoskeleton & 3 & $4.26 E-03$ \\
\hline GO:0043228 & Non-membrane-bounded organelle & 6 & $5.00 E-03$ \\
\hline GO:0043232 & Intracellular non-membrane-bounded organelle & 6 & $5.00 E-03$ \\
\hline GO:0001533 & Cornified envelope & 2 & $1.20 E-02$ \\
\hline GO:0018149 & Peptide cross-linking & 2 & $1.71 E-02$ \\
\hline GO:0031424 & Keratinization & 2 & $2.82 E-02$ \\
\hline
\end{tabular}

GO: Gene Ontology; DEGs: differentially expressed genes.

total of 100 significant genes were obtained from GSEA, and the genes with positive correlation were plotted. GSEA analysis, including FABP5, IVL, KRT6A, KRT15, KRT16, and $T I M P 2$, suggested that the most involved hallmarks included the $P 53$ pathway, K-ras signaling, estrogen response late, and estrogen response early. The details are illustrated in Figure 8.

\section{Discussion}

Melanoma is an aggressive and devastating cancer directly derived from melanocytic nevus, characterized by a typically high incidence in people with the $B R A F^{V 600 E / K}$ mutation [33]. Alterations in the activity of the MAPK signal transduc- tion pathway are associated with metastasis in melanoma patients $[34,35]$. Specifically, constitutively activated components of the MAPK pathway serve as targets for therapy of melanoma [5]. However, available evidence is insufficient for developing management or diagnostic strategies to improve the survival of patients with melanoma. For example, a study of the expressions of $P D-L 1, P D-L 2, P D-1$, and $C Y T$ in melanomas found that mutation density contributes significant prognostic value [36]. Unfortunately, most patients with melanoma, which are initially diagnosed with highly aggressive and progressive disease, are therefore not candidates for curative therapies [2]. Hence, highly effective biomarkers for diagnosis and treatment are urgently required. 


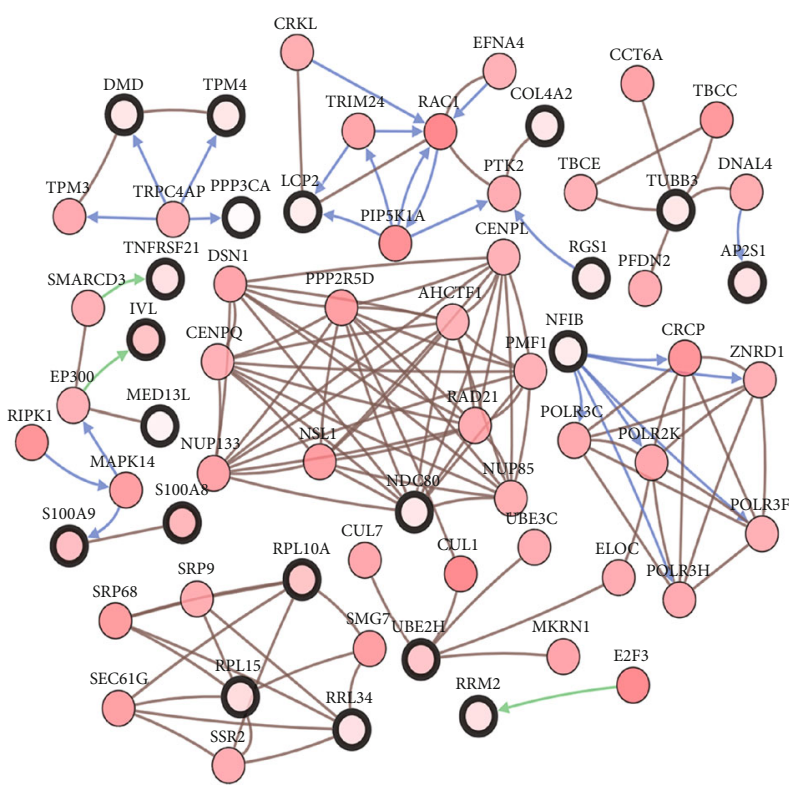

(a)

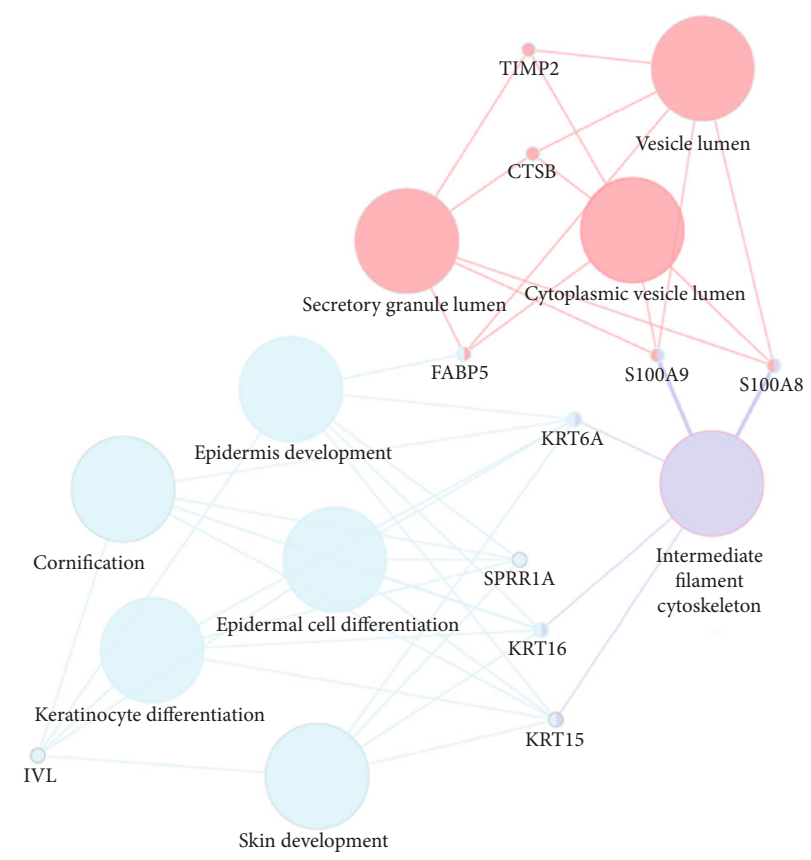

(b)

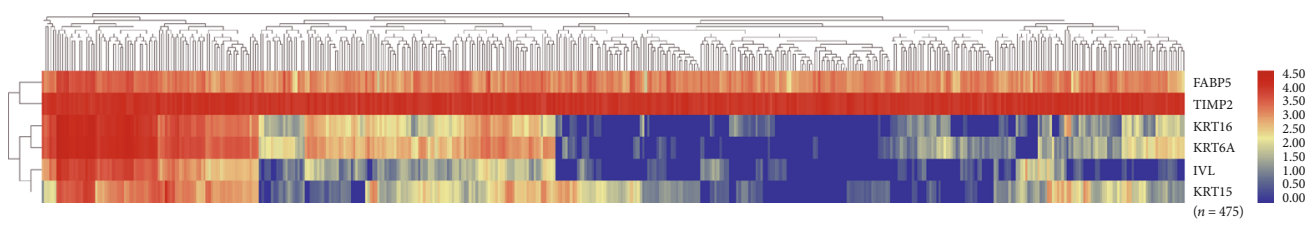

(c)

Figure 3: Interaction network and biological process analysis of the hub genes. (a) Hub genes and their coexpression network were analyzed by using cBioPortal. Nodes with bold black outline represent hub genes. Nodes with thin black outline represent the coexpression genes. (b) The biological process analysis of hub genes was constructed by using ClueGO. Different colors of nodes refer to the functional annotation of ontologies. Corrected $p$ value $<0.01$ was considered statistically significant. (c) Hierarchical partitioning of 6 significant hub genes was obtained from DNA microarrays $(n=475)$. It represented the level of expression of 6 genes across a number of comparable samples with high expression samples marked in red and low in blue.

Aberrant genetic and epigenetic regulation of key metabolic pathways is known to contribute towards the development and progression of CM. Here, our analysis of the expression profiles of melanocytic nevus and melanomas identified 73 DEGs and 10 hub genes, as well as the functional enrichment and significant pathways. GO and KEGG enrichment indicated significant associations with the terms ectoderm development, epidermis development, negative regulation of signal transduction, and negative regulation of cell communication. In addition, GSEA analysis showed that the most involved hallmarks included the $P 53$ pathway, $K$-ras signaling, estrogen response late, and estrogen response early. Increasing evidence supports a role for P53 in the progression of melanoma, particularly in NRAS-driven melanomas, which grow more aggressively compared with those with BRAF mutations [37]. P53 mutations occur in $15 \%$ of melanomas with NRAS mutations [37]. We suggest therefore that reactivating P53 expression in melanoma may be potentially important for enhancing therapy. Constitutive activation of KRAS stimulates cell proliferation and inhibits apoptosis. Increasing evidence showed that KRAS mutations are closely associated with multiple cancers, including non-small-cell lung cancer, colorectal cancer, and pancreatic cancer, and NRAS mutations are present in melanomas [38]. Therefore, role of KRAS signaling in melanoma must be evaluated. Moreover, evidence shows significant protective roles for estrogen signaling in the pathogenesis and progression of melanoma [39]. Although the mechanism is unknown, this clinical association suggests the importance of estrogen signaling in the progression of the malignant phenotype of melanoma.

Further, among the 6 prognostic hub genes, differential expressions of the genes encoding fatty acid-binding protein 5 (FABP5); involucrin (IVL); keratins 6A, 15, and 16 (KRT6A, KRT15, and KRT16); and tissue inhibitor of metalloproteinases 2 (TIMP2) was significantly associated with prognosis of patients with melanoma based on the survival analysis and COX models.

FABP5 plays an important role in binding free fatty acids, as well as in the regulation of lipid metabolism and transport. Urinary excretion of FABP5 is detected in patients with stages II and III cutaneous melanoma but not those with 


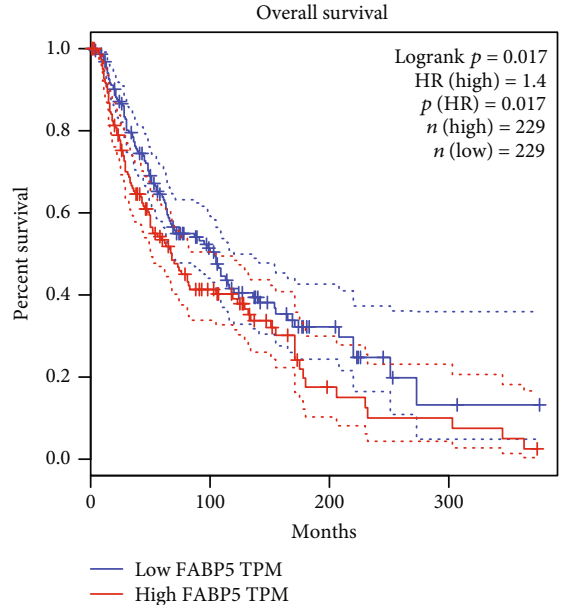

(a)

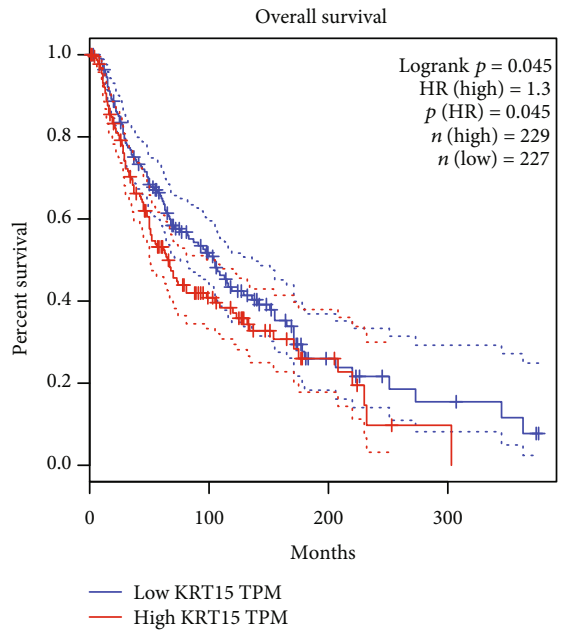

(d)

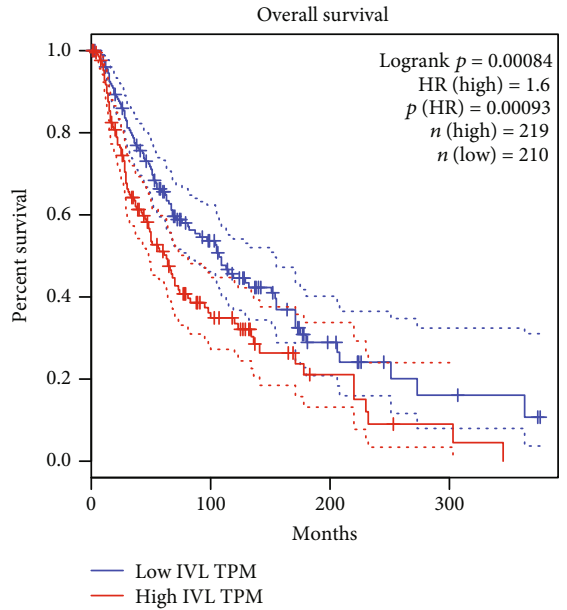

(b)

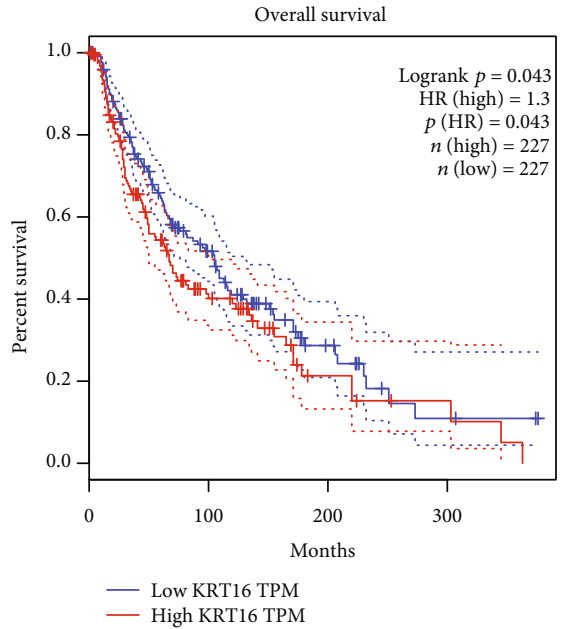

(e)

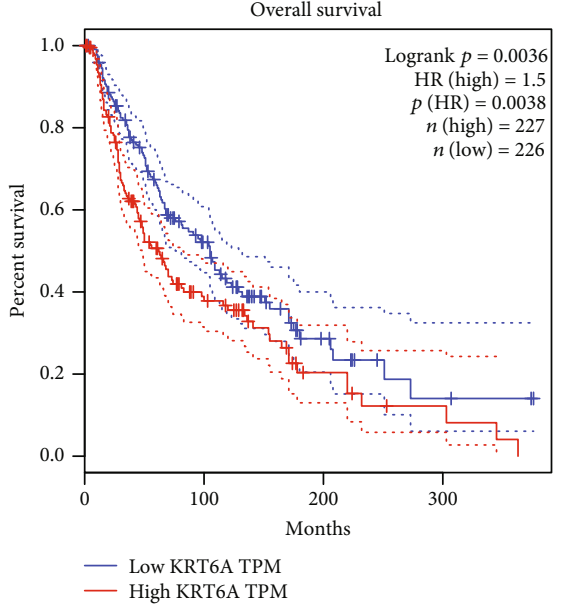

(c)

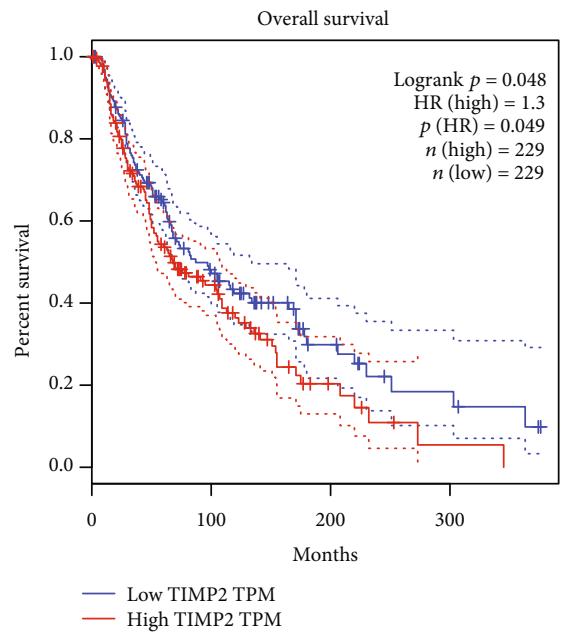

(f)

FIGURE 4: Univariate survival analysis of the hub genes was performed using the Kaplan-Meier curve. The horizontal axis ( $x$-axis) represented time in days, and the vertical axis ( $y$-axis) showed the probability of surviving or the proportion of people surviving. The dotted lines represented the $95 \%$ confidence interval information in the survival plot, with high expression marked in red and low expression marked in blue. The lines presented survival curves of two groups. Each elevated expression in 6 hub genes showed markedly significant worse OS in melanoma samples $(p<0.05)$.

stage IV melanoma [40]. Insufficient information is available concerning the role of FABP5 in melanomas vs. its association with prognosis $[41,42]$. In the present study, FABP5 was found with significant prognostic value not only in survival analysis by GEPIA but also in multivariate Cox models by BACK-LR methods. Hence, further research is required to confirm our hypothesis.

IVL is a component of the human skin that contributes to the formation of the envelope that protects corneocytes [43]. IVL is produced in a free form during the early stages of the terminal differentiation of keratinocytes. Increased expression of IVL correlates with undesirable outcomes of squamous cell carcinoma [44], and IVL contributes to inflammatory skin diseases such as psoriasis [45]. Our present findings therefore likely will encourage further investigations of the clinical significance of IVL in human disease, although few reports address its value for predicting the prognosis of melanoma.
Keratin (KRT), which is a constituent of the hair, nails, and the outer layer of the human skin, protects epithelial cells from damage or pressure [46]. The gene encoding KRT6A, which is located within the type II keratin gene cluster on human chromosome 12q [47], is closely associated with the prognosis and diagnosis of lung and breast cancers; however, few studies address its role in melanoma [48, 49]. In our study, KRT6A showed significant prognostic value among all the hub genes according to the multivariate Cox models by BACK-LR methods, indicating it might act unique role in CM patients' survival outcomes. The type I cytokeratin KRT15 is expressed by certain progenitor basal cells within the epithelium [50]. For example, KRT15 is present in the adult and fetal human skin of hair-bearing, non-hair-bearing, and palmoplantar regions and is coordinately expressed with melanoma-associated chondroitin sulfate proteoglycan [51]. The type I cytokeratin KRT16 forms a complex with 


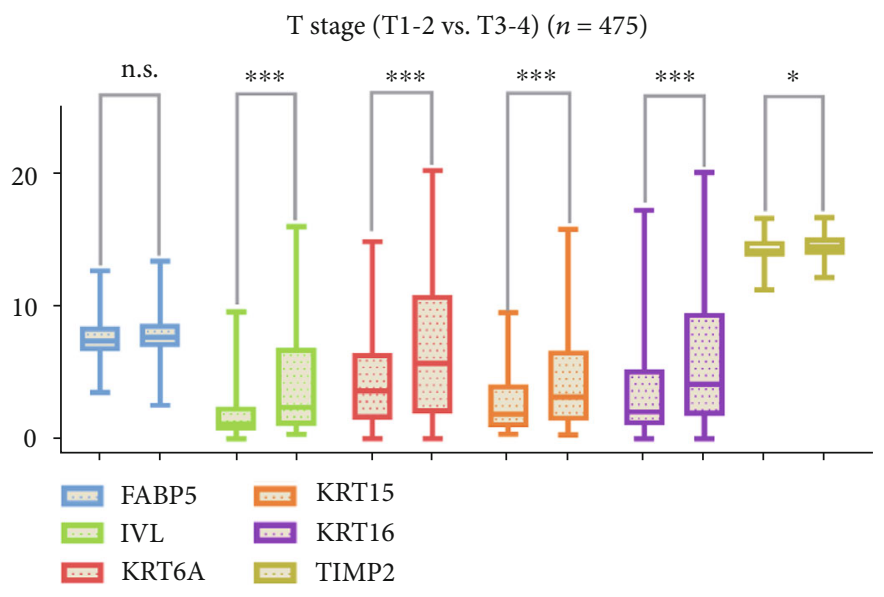

(a)

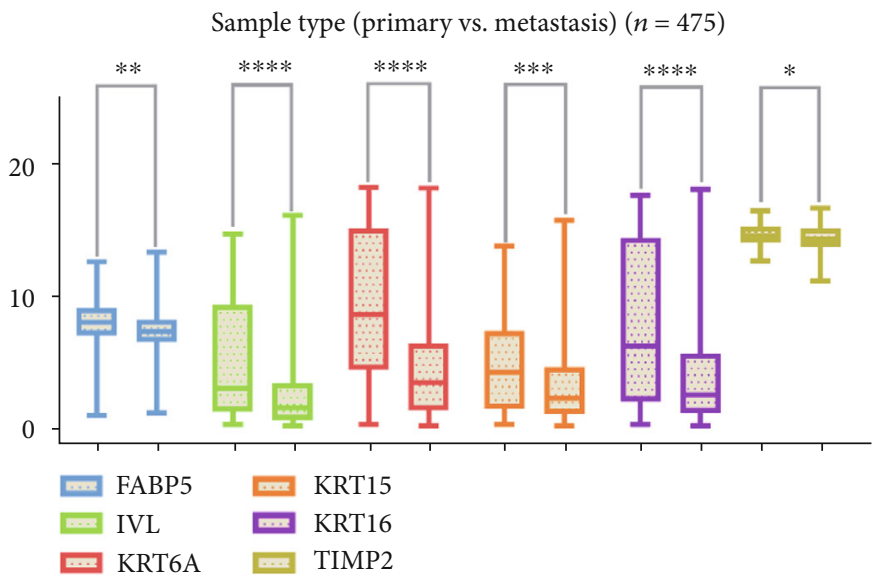

(b)

FIGURE 5: (a) Except FABP5, elevated expression patterns of $I V L, K R T 6 A$, KRT15, KRT16, and TIMP2 were significantly associated with T stage (T1-T2 vs. T3-T4) $(n=475)$. (b) Six hub genes are highly expressed in primary than metastatic sites $(n=475)$ (FABP5 presents in light blue, IVL in light green, KRT6A in red, KRT15 in orange, KRT16 in purple, and TIMP2 in dark green).

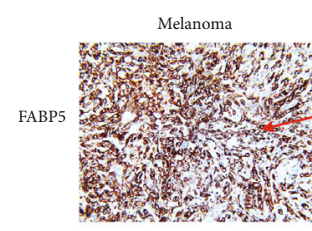

Nevus
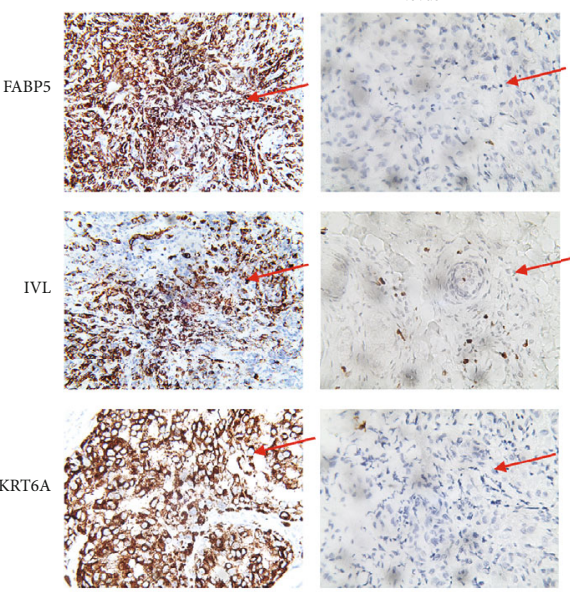
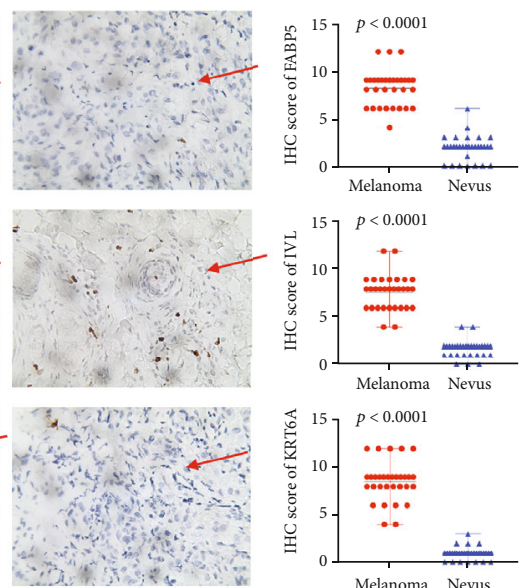
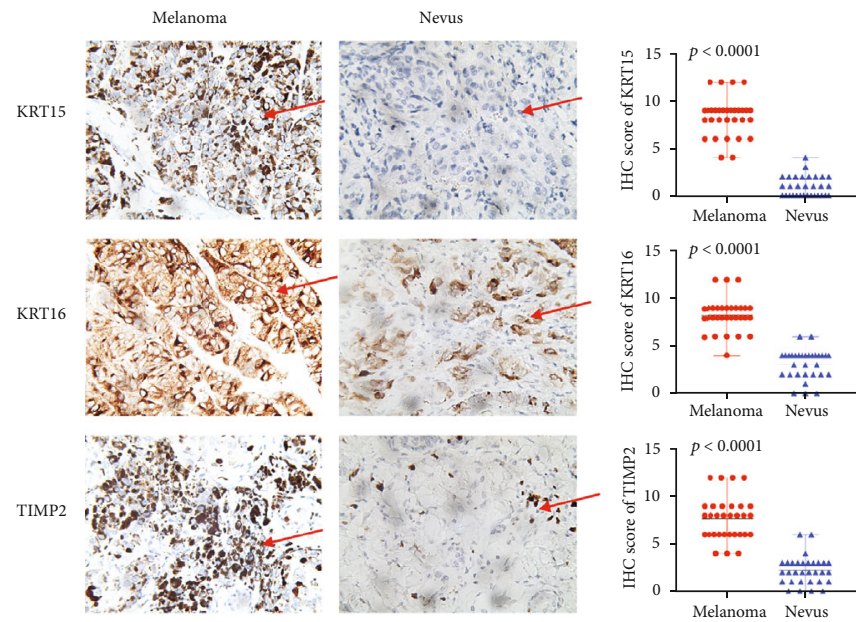

FIGURE 6: (a) IHC staining indicated significantly elevated FABP5, IVL, KRT6A, KRT15, KRT16, and TIMP2 expressions in terms of density and intensity in melanoma tissues compared with nevus tissues. All melanocyte contents were shown in red arrows. (b) Scatter plots of IHC score between melanoma and nevus tissues were illustrated $(p<0.0001)$. 


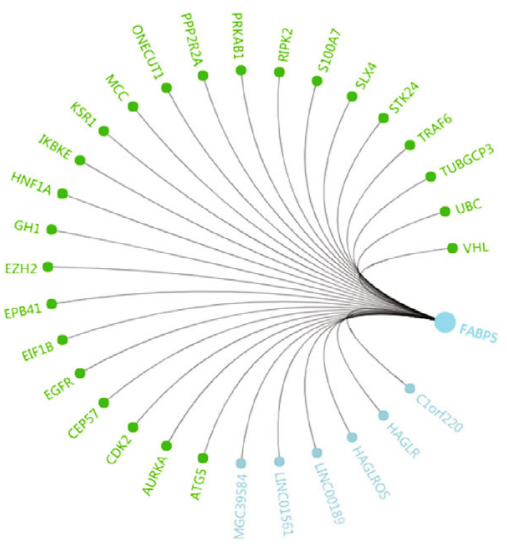

Transcription factor regulation-DNA binding
Transcription factor regulation-activation
Related lncRNA
Targeted miRNA
Protein-protein interaction

(a)

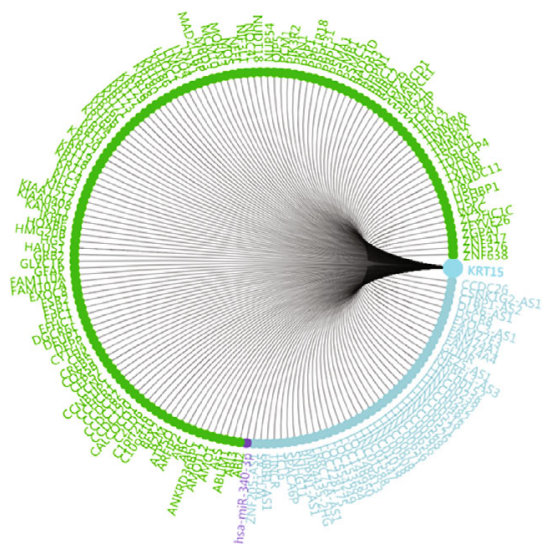

Transcription factor regulation-DNA binding
Transcription factor regulation-activation
Related lncRNA
Targeted miRNA
Protein-protein interaction

(d)

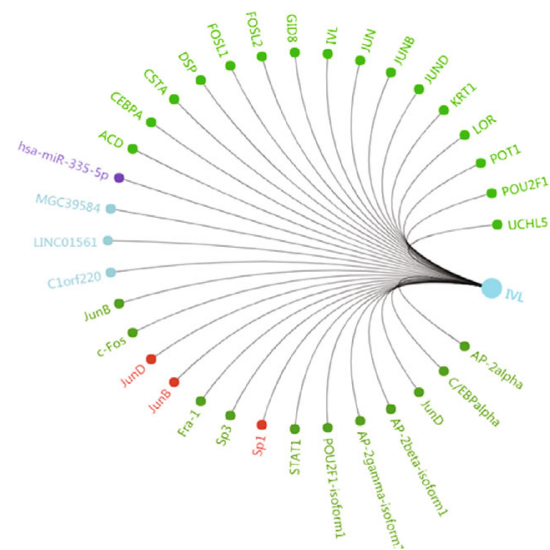

Transcription factor regulation-DNA binding
Transcription factor regulation-activation
Related lncRNA
Targeted miRNA
Protein-protein interaction

(b)

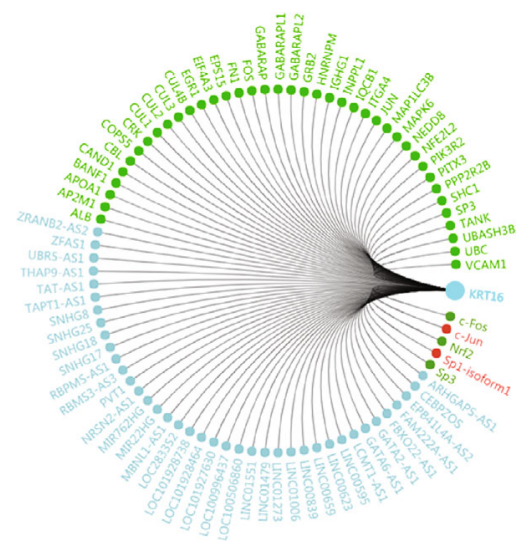

Transcription factor regulation-DNA binding
Transcription factor regulation-activation
Related lncRNA
Targeted miRNA
Protein-protein interaction

(e)

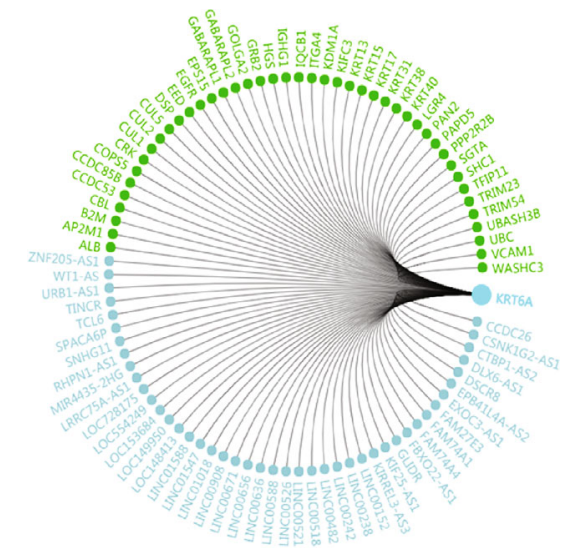

Transcription factor regulation-DNA binding Transcription factor regulation-activation Related lncRNA

Targeted miRNA

Protein-protein interaction

(c)

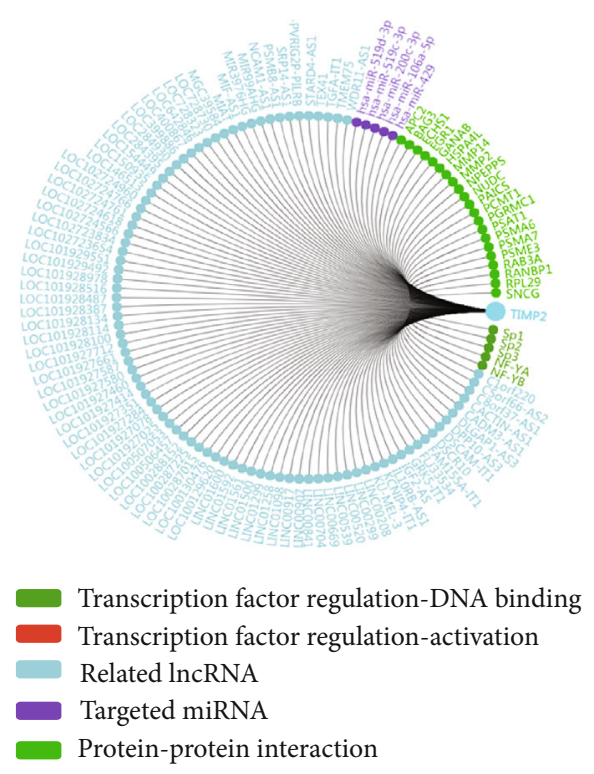

(f)

FiguRe 7: Transcription factor regulation network was constructed in FABP5 (a), IVL (b), KRT6A (c), KRT15 (d), KRT16 (e), and TIMP2 (f). Significant nodes were marked in different colors in line with hub genes (transcription factor regulation-DNA binding, transcription factor regulation-activation, related lncRNA, targeted miRNA, and protein-protein interaction).

KRT6 in numerous epithelial tissues. Mutations in KRT6 are associated with hereditary skin diseases, and KRT16 contributes to the immune response to tumors and in tumor cell development [52]. Moreover, the levels of KRT16 expression may discriminate metastasis from primary melanoma [53]. These findings demonstrate the significant role of keratins in melanoma.

Evidence indicates that TIMP2 functions as a suppressor of metastasis. For example, elevated expression of TIMP2 suppresses the proliferation of melanoma cells via the Wnt/?catenin signal transduction pathway, indicating that TIMP2 contributes to the pathogenesis and progression of melanoma
[54]. In the current study, TIMP2 was found elevated in primary CM, which is consistent with previous studies. Further studies are needed to elucidate underlying potential carcinogenesis progressive nevus and melanoma.

This study is the first to our knowledge that has attempted to construct a gene regulatory network incorporating DEGs between nevus and melanoma, as well as to functionally annotate hub genes in melanoma. Further, alterations in the expression profiles of FABP5, IVL, KRT6A, KRT15, KRT16, and TIMP2 were significantly associated with worse prognosis, indicating that these hub genes may participate in the aggressive transformation from a nevus to malignant melanoma. While 


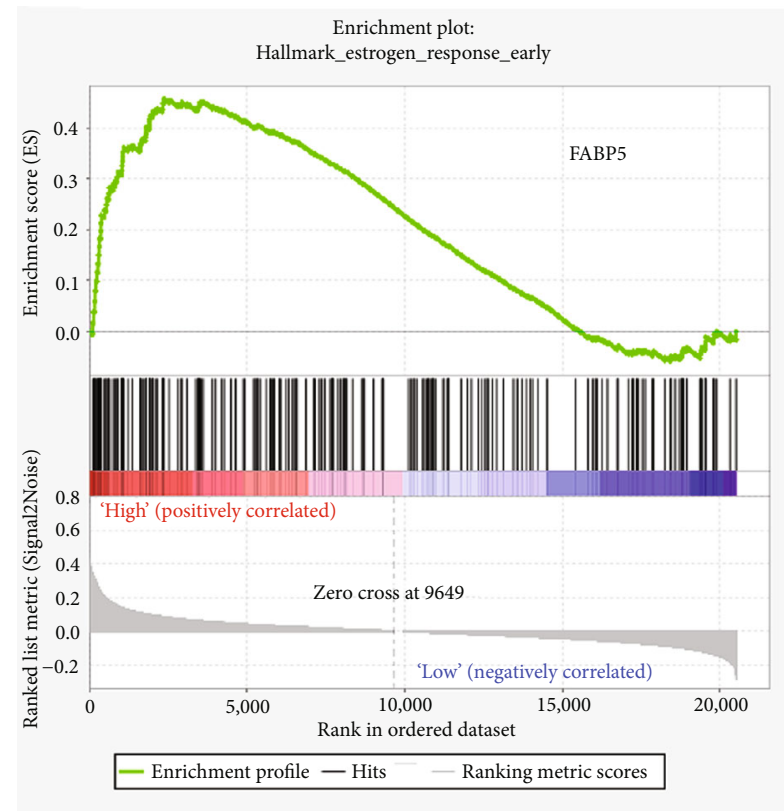

(a)

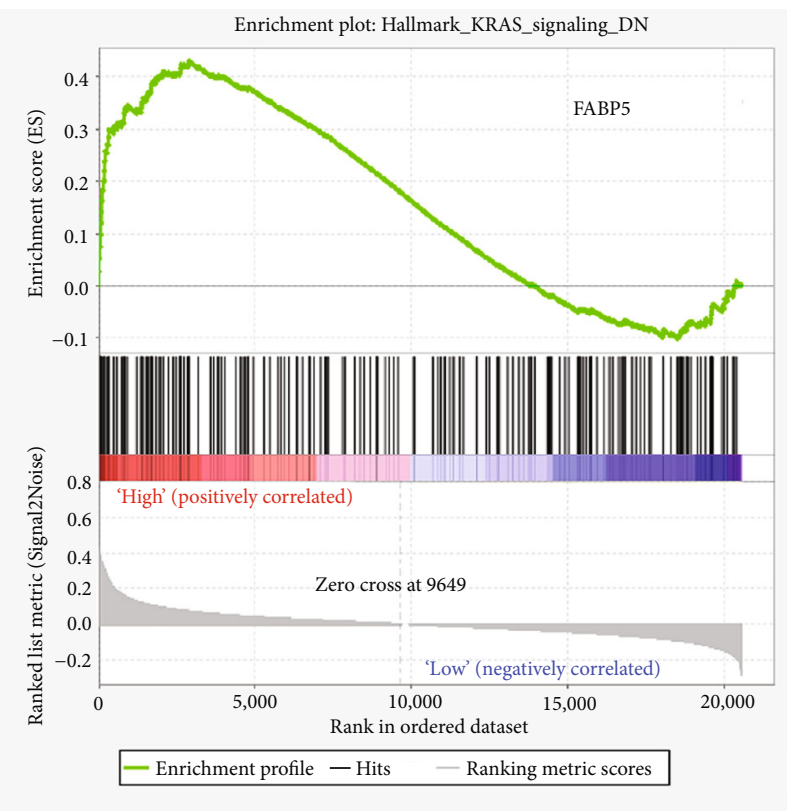

(c)

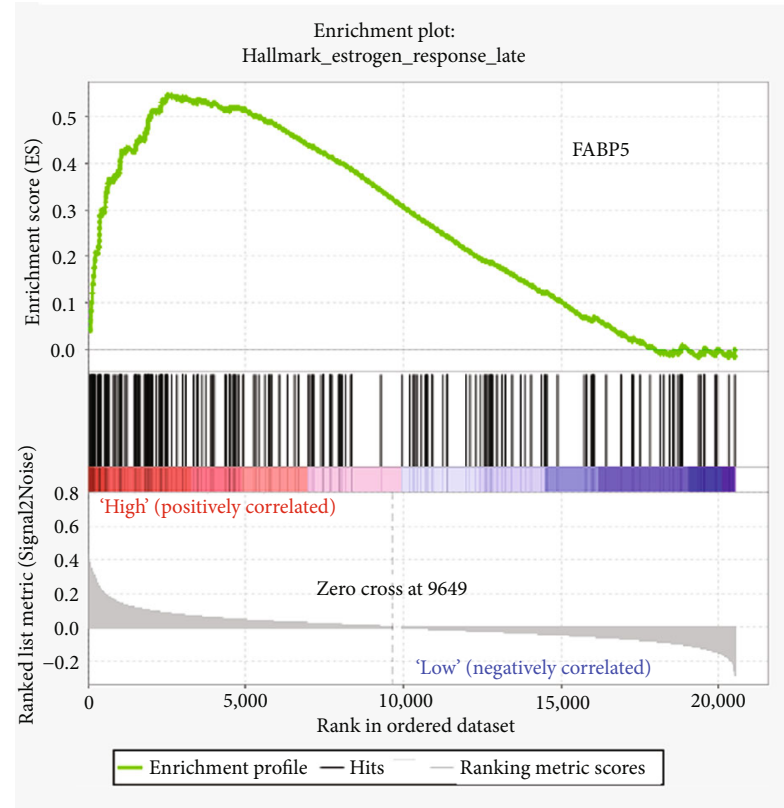

(b)

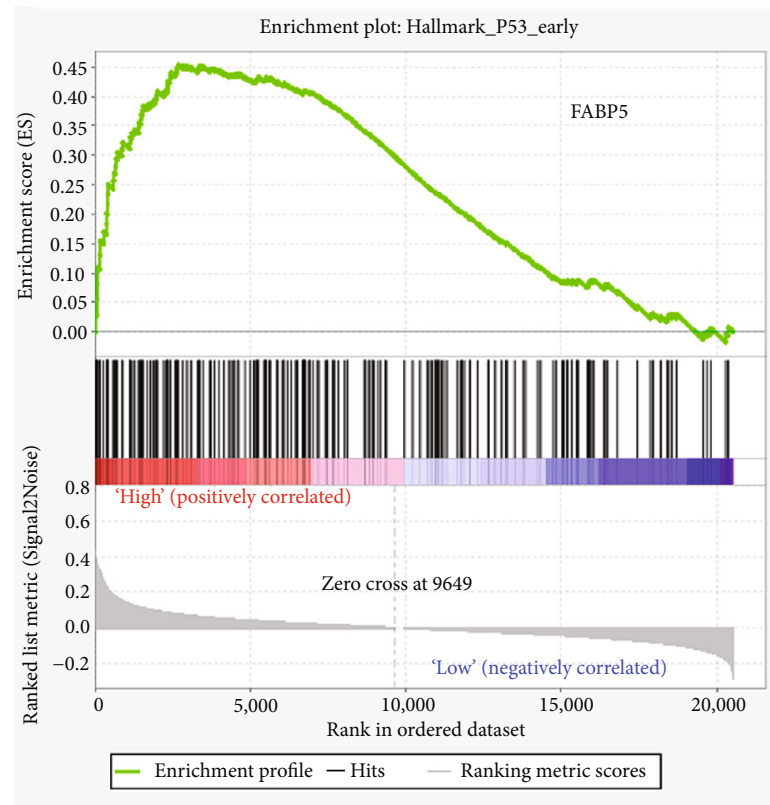

(d)

Figure 8: Continued. 


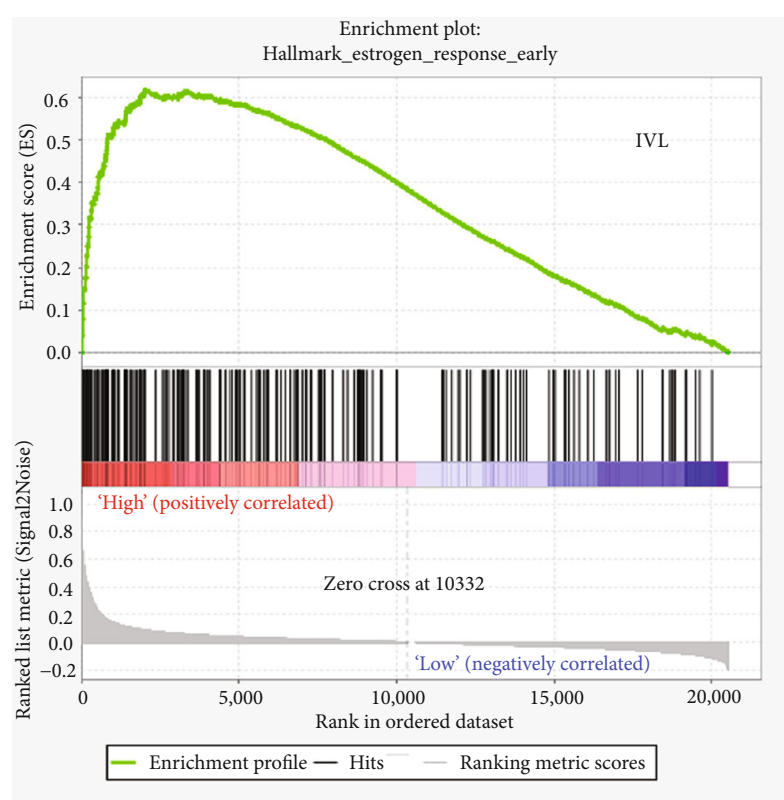

(e)

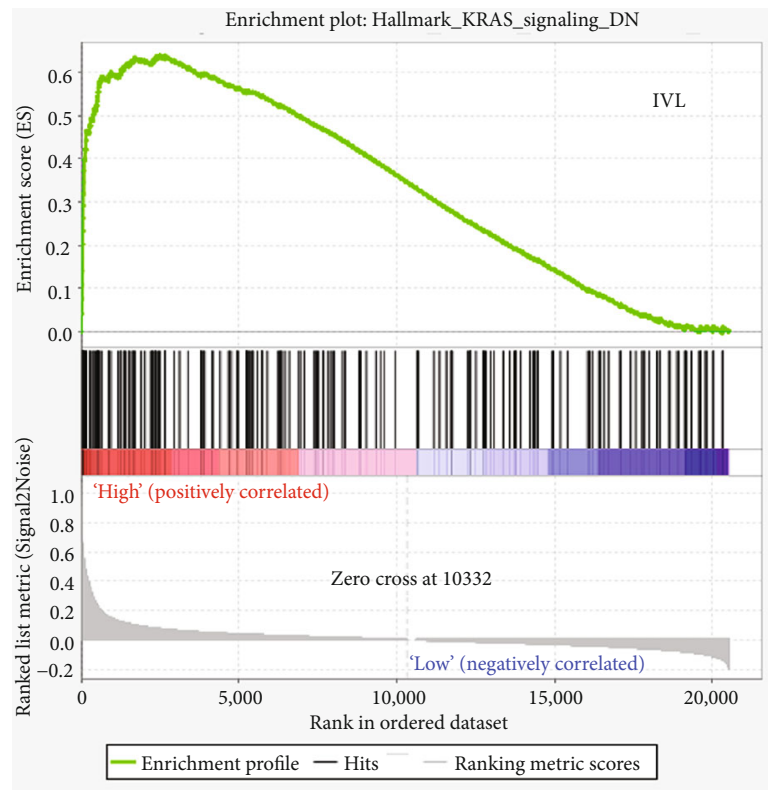

(g)

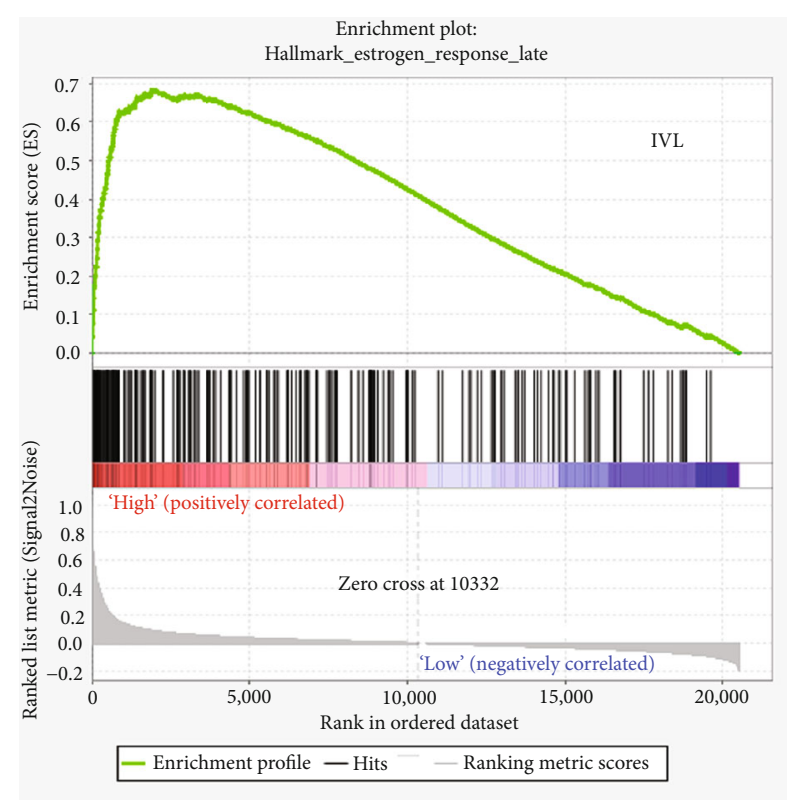

(f)

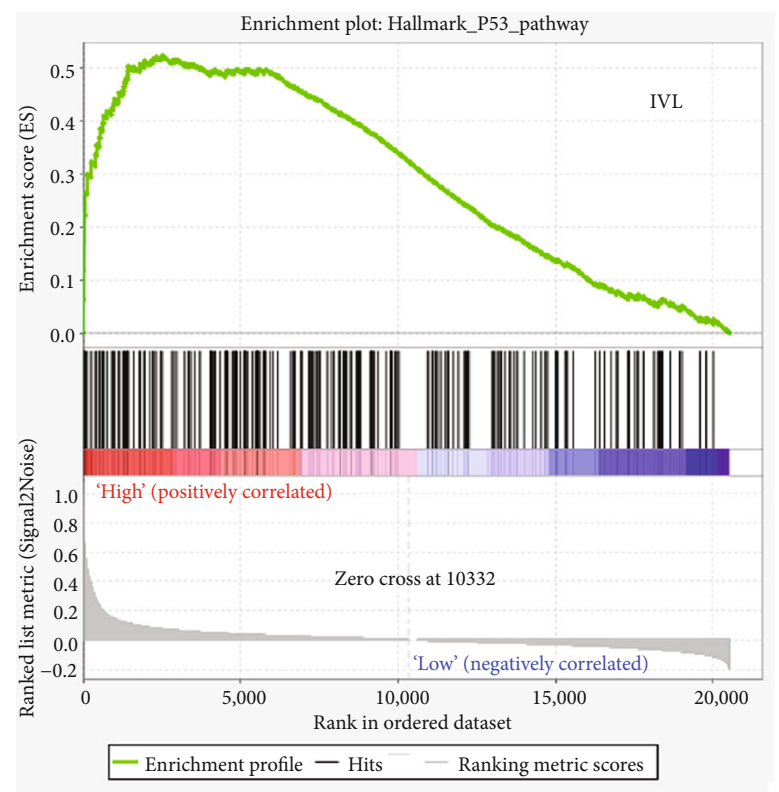

(h)

Figure 8: Continued. 


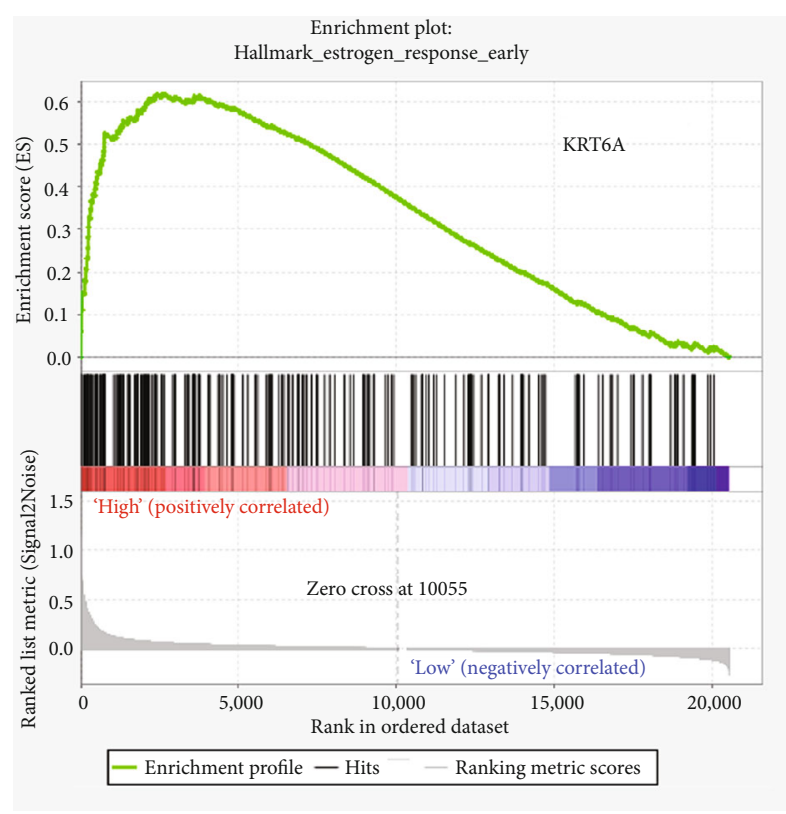

(i)

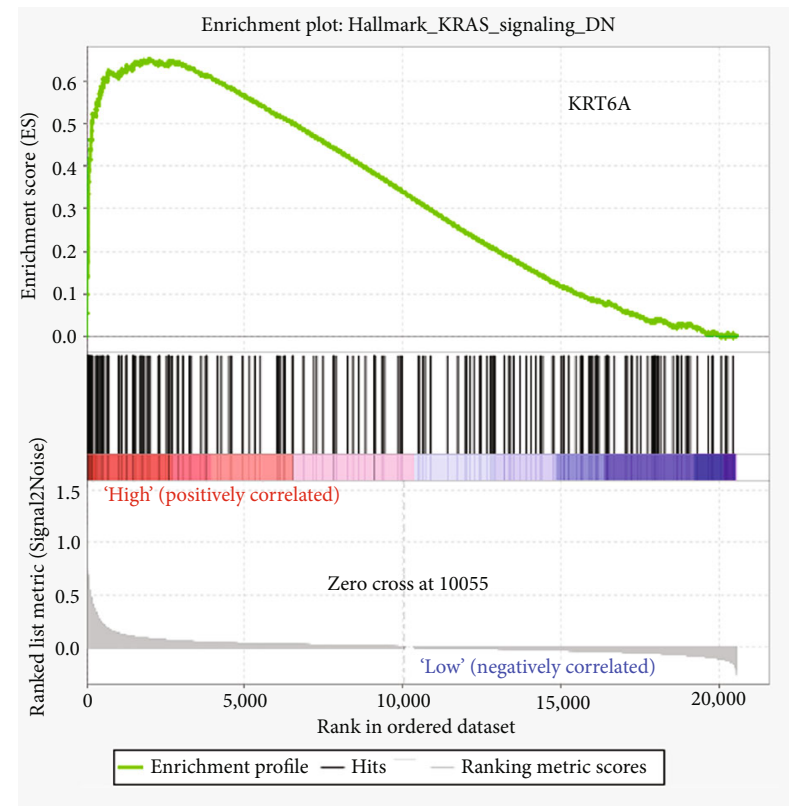

(k)

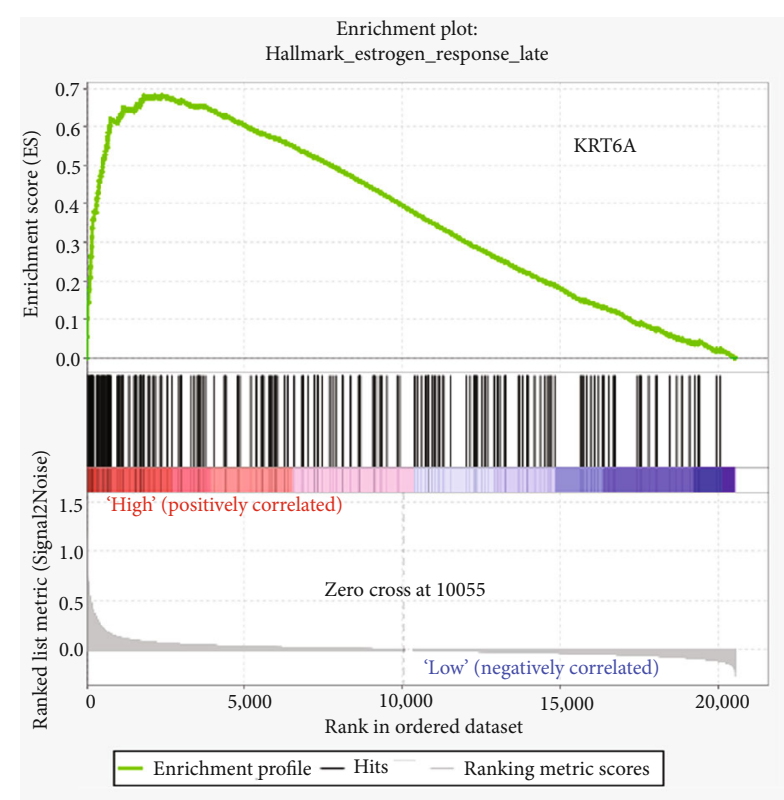

(j)

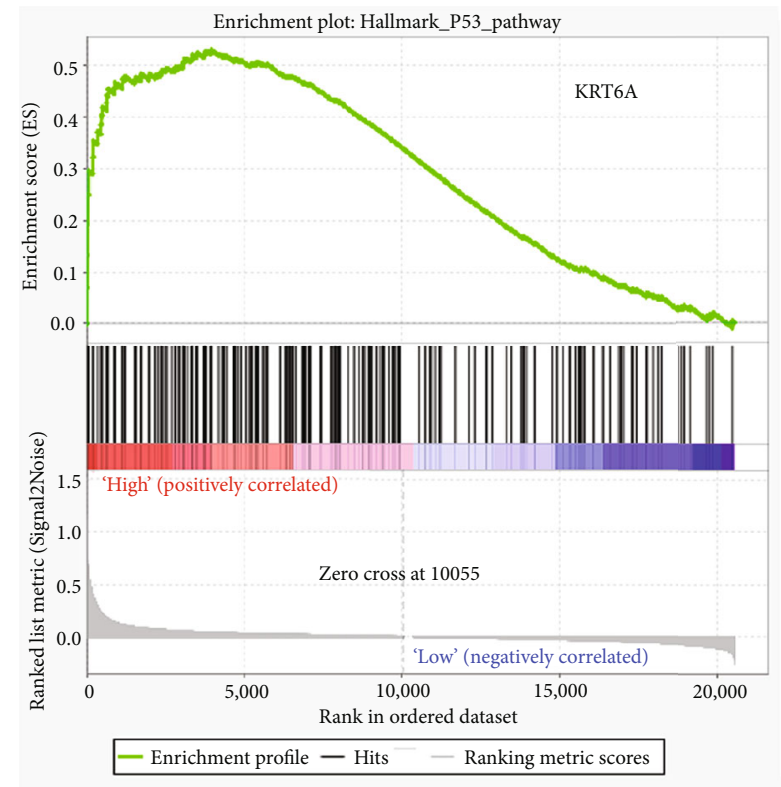

(l)

Figure 8: Continued. 


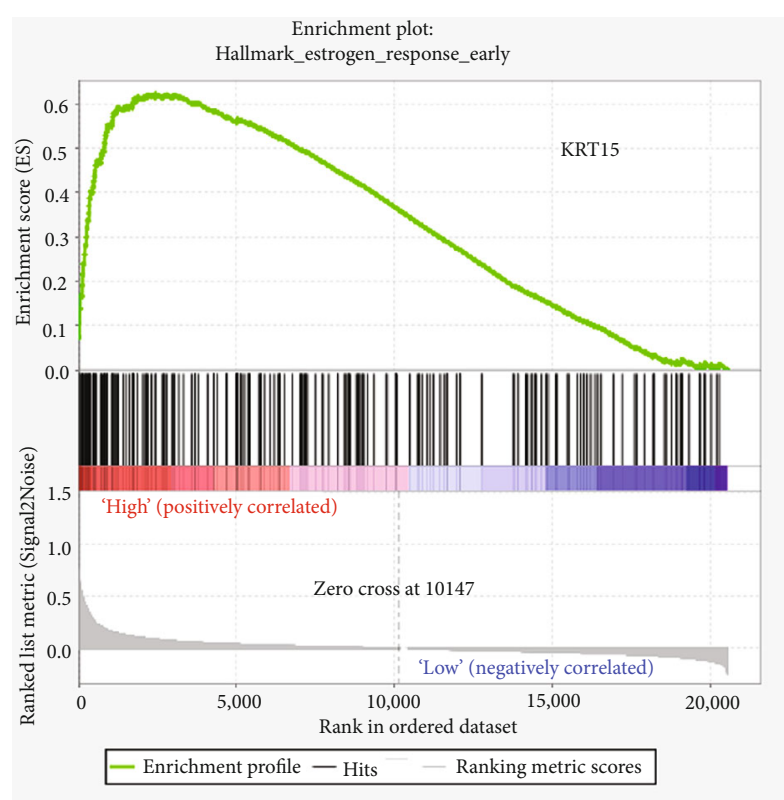

(m)
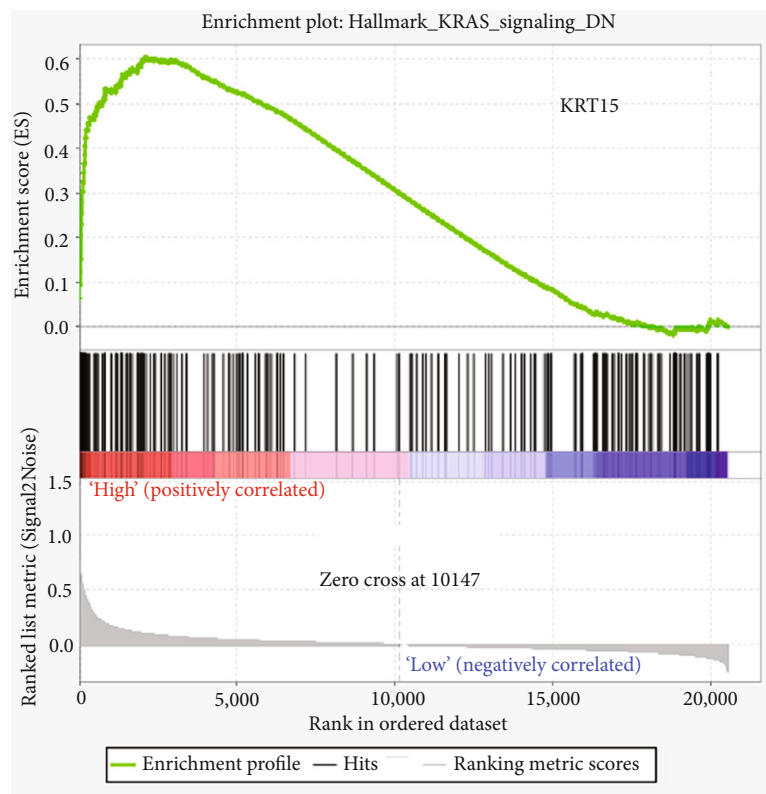

(o)

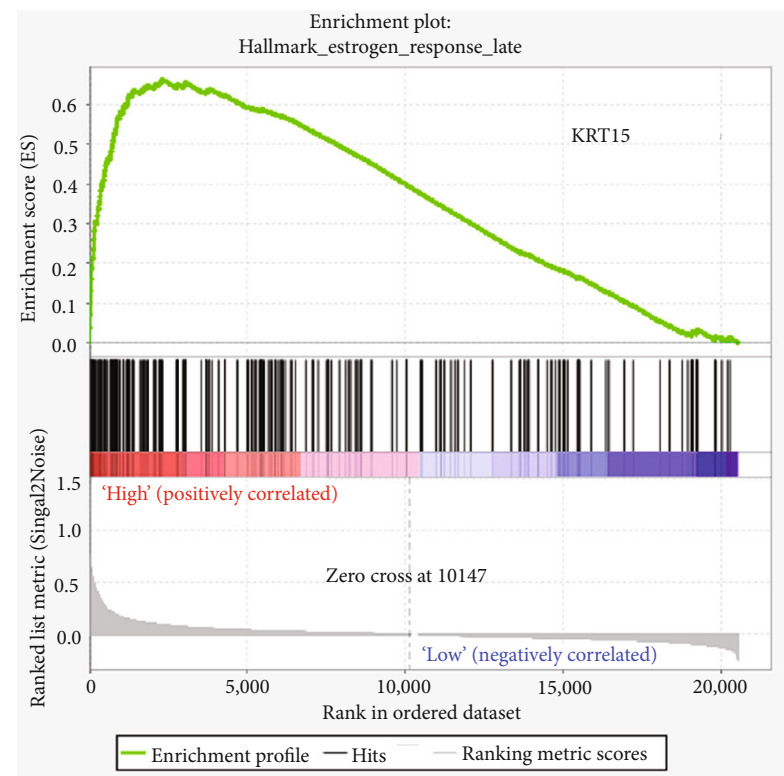

(n)

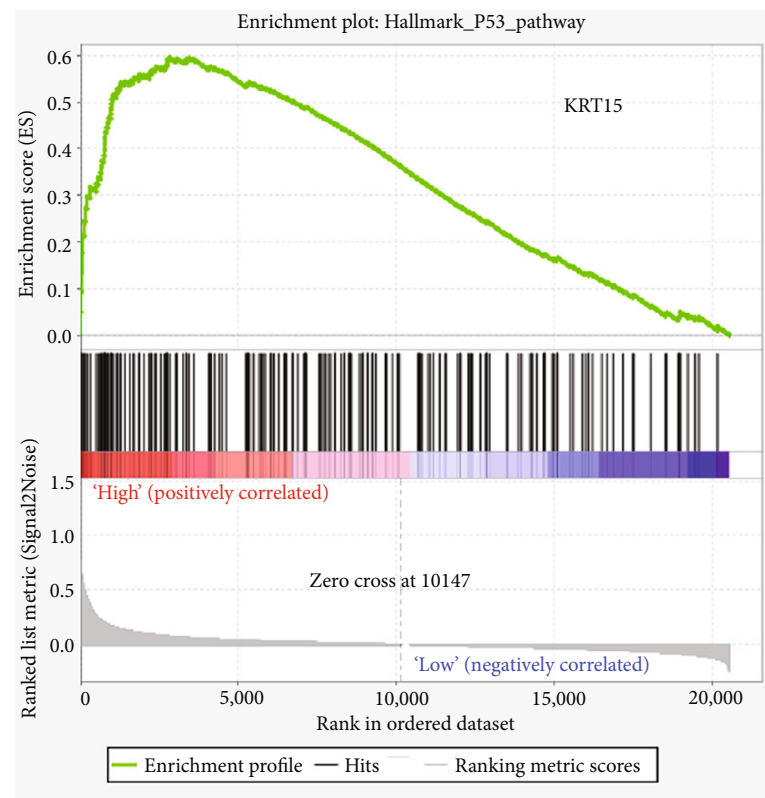

(p)

Figure 8: Continued. 


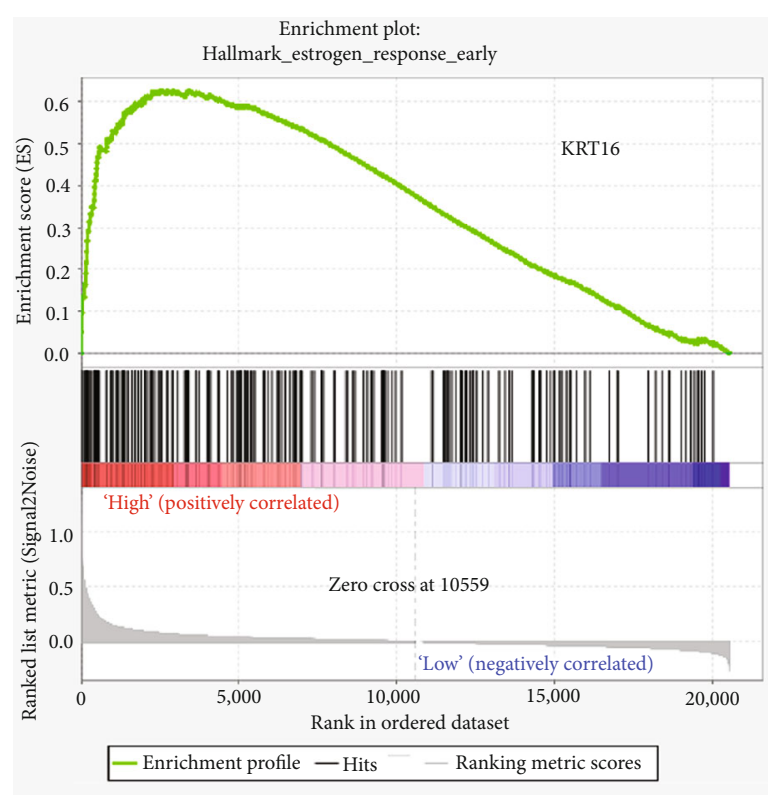

(q)

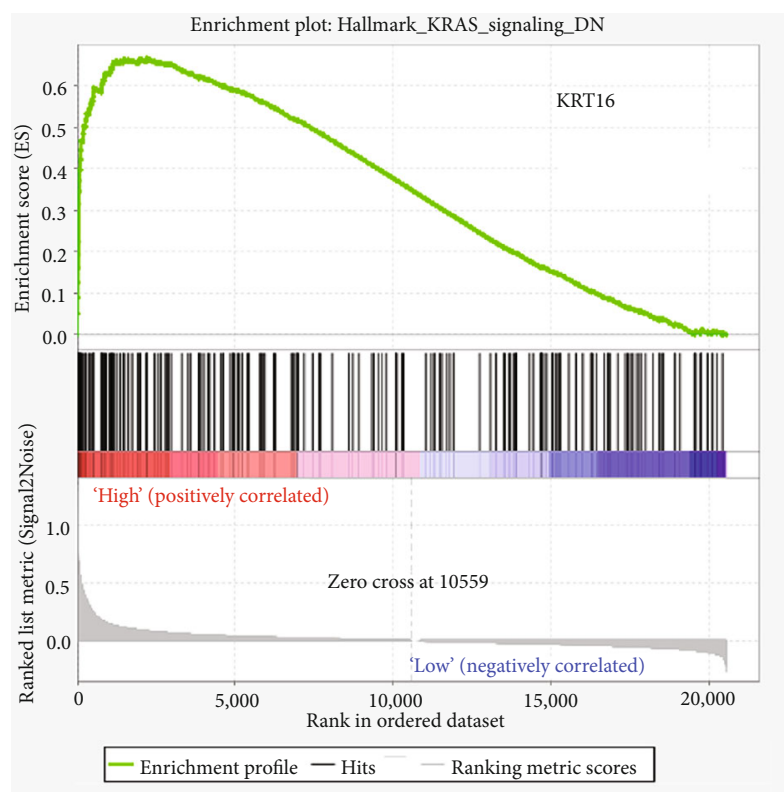

(s)

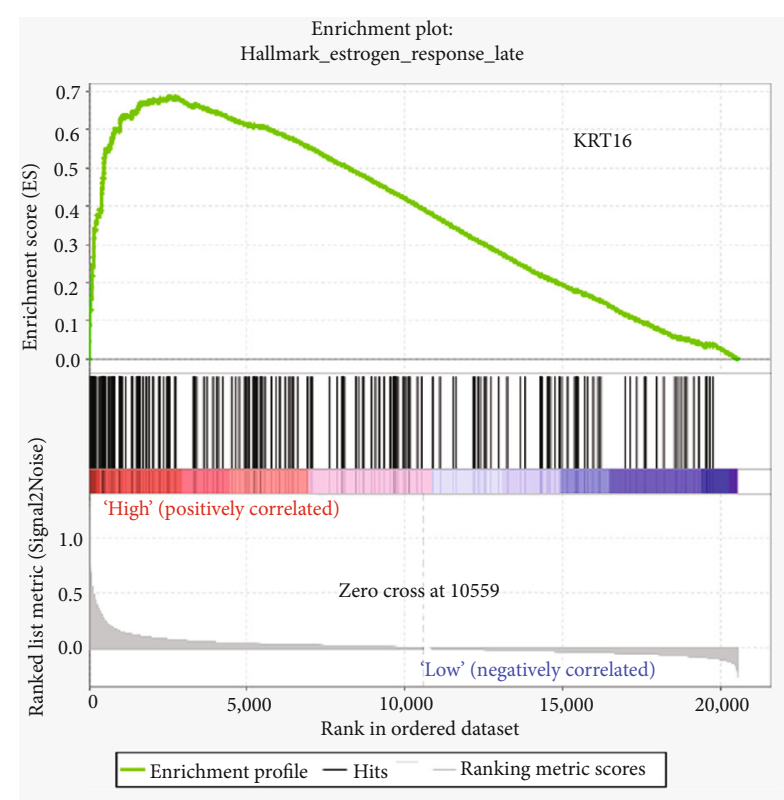

(r)

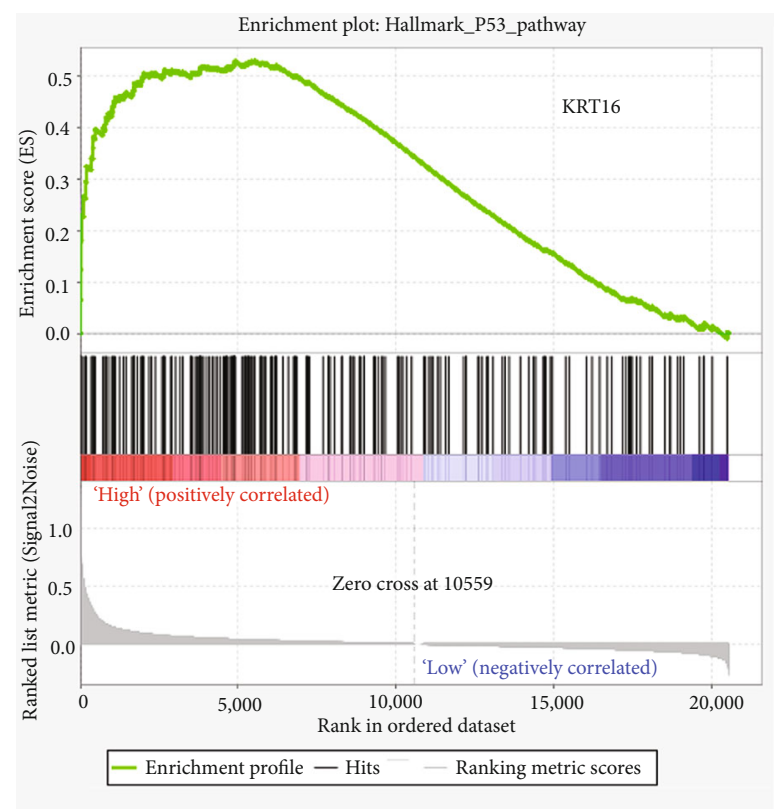

$(\mathrm{t})$

FIgUre 8: Continued. 


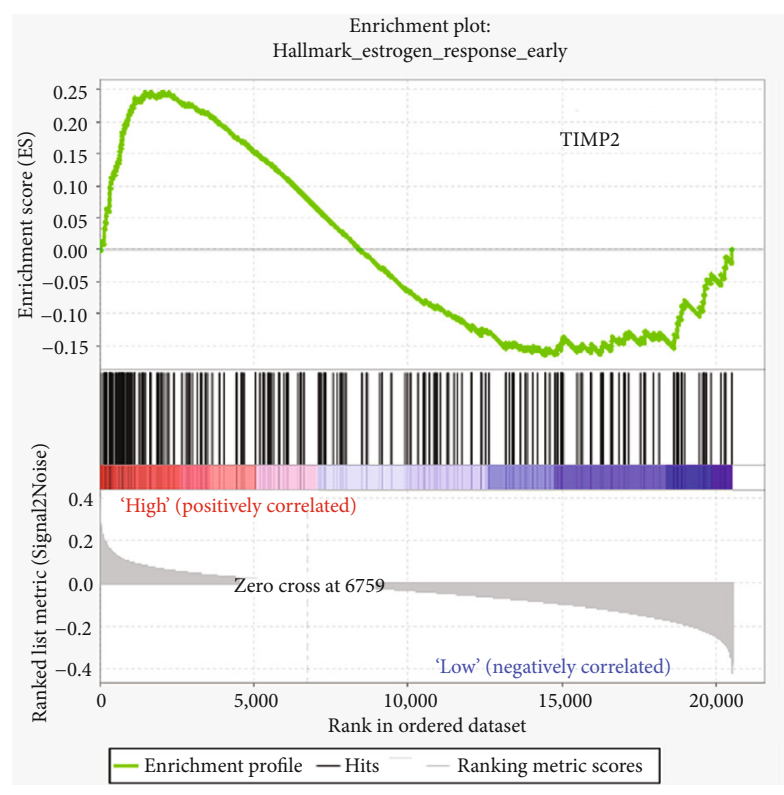

(u)

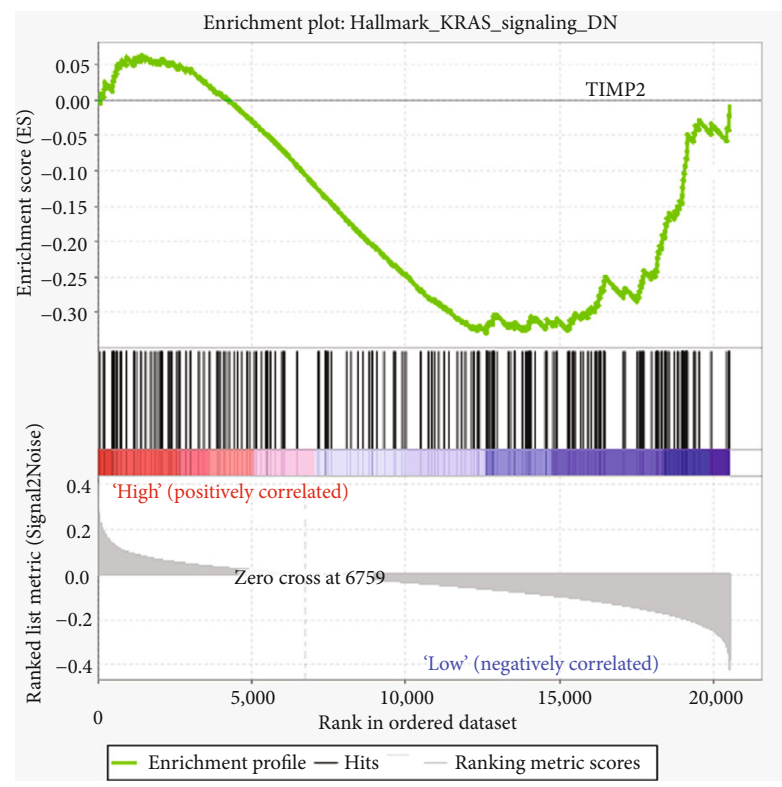

(w)

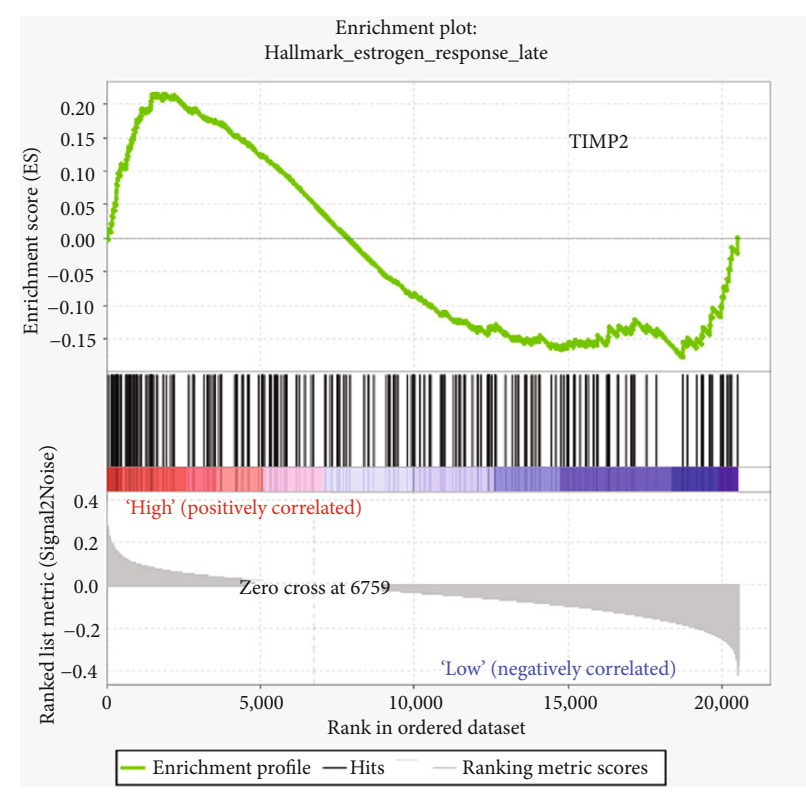

(v)

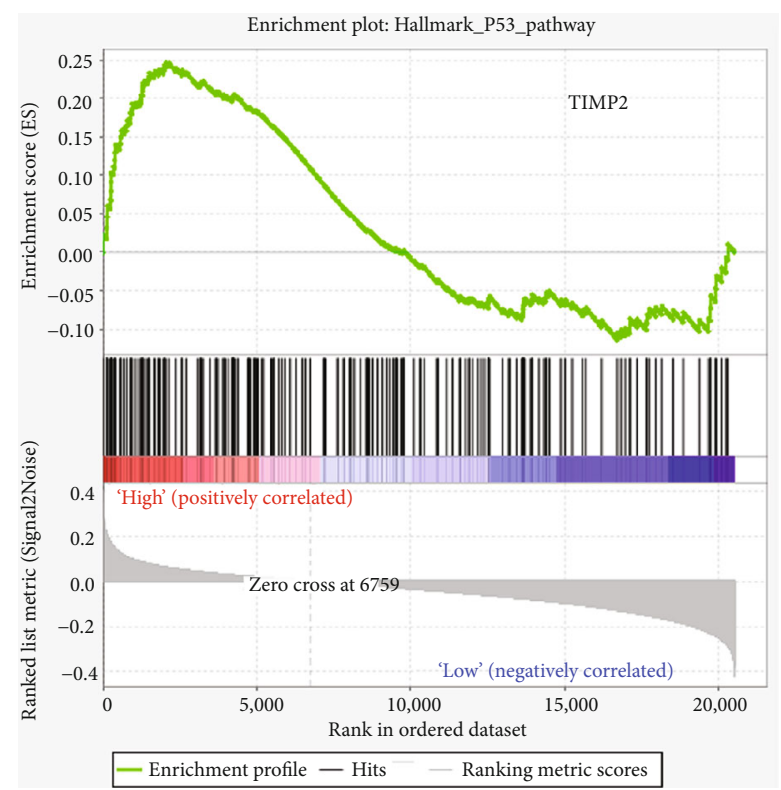

(x)

Figure 8: A total of 100 significant genes were obtained from GSEA with positive and negative correlations. GSEA was used to perform hallmark analyses in FABP5, IVL, KRT6A, KRT15, KRT16, and TIMP2, respectively. The most involved significant pathways included the $\mathrm{P} 53$ pathway, estrogen response late, estrogen response early, and K-ras signaling.

merely expression level of hub genes was identified in this study, thus further functional works, as well as validated cohorts, were needed to verify the absoluteness of these findings.

\section{Conclusion}

In summary, this study identified significant DEGs participating in the process of malignant transformation from nevus to melanoma tissues. Expression profiles of FABP5, IVL, KRT6A, KRT15, KRT16, and TIMP2 provide clues of prognostic implications, which might help us evaluate malignant potential of nevus and underlying carcinogenesis progress from melanocytic nevus to melanoma. These hub genes are associated with major biological pathways such as the $P 53$ pathway, $K$-ras signaling, estrogen response late, and estrogen response early. However, further studies are required to elucidate molecular pathogenesis and alteration in signaling pathways of these hub genes in melanoma.

\section{Data Availability}

The datasets analyzed for this study can be found in the GEO (https://www.ncbi.nlm.nih.gov/geo) and TCGA (https:// www.cancer.gov). 


\section{Conflicts of Interest}

The authors declare that the research was conducted in the absence of any commercial or financial relationships that could be construed as a potential conflict of interest.

\section{Authors' Contributions}

The work presented here was carried out in collaboration among all authors. SGL, ZXY, and ZHL defined the research theme, discussed analysis, interpretation, and presentation. HW drafted the manuscript, analyzed the data, developed the algorithm, and interpreted the results. XWH and WJX coworked on associated data collection and performed IHC. HJY helped to perform the statistical analysis and reference collection. All authors read and approved the final manuscript. Wei Han, Wen-Hao Xu, and Jian-Xiong Wang contributed equally to this work.

\section{Acknowledgments}

We thank Liwen Bianji, Edanz Editing China (http://www .liwenbianji.cn/ac), for editing the English text of a draft of this manuscript.

\section{Supplementary Materials}

Supplementary Figure 1: functional and pathway enrichment analyses were performed using DAVID in bubble chart. Supplementary Figure 2: a network of the 10 genes and their coexpression genes was visualized and displayed in detail. Supplementary Table 1: gene symbols of 73 DEGs. Supplementary Table 2: multivariate Cox regression analysis of OS and PFS in TCGA cohort using BACK-LR methods (OS: overall survival; PFS: progression-free survival; TCGA: The Cancer Genome Atlas). (Supplementary Materials)

\section{References}

[1] R. L. Siegel, K. D. Miller, and A. Jemal, "Cancer statistics, 2020," CA: a Cancer Journal for Clinicians, vol. 70, no. 1, pp. 7-30, 2020.

[2] D. Schadendorf, A. C. J. van Akkooi, C. Berking et al., "Melanoma," The Lancet, vol. 392, no. 10151, pp. 971-984, 2018.

[3] A. Mohammadpour, M. Derakhshan, H. Darabi, P. Hedayat, and M. Momeni, "Melanoma: where we are and where we go," Journal of Cellular Physiology, vol. 234, no. 4, pp. 33073320, 2019.

[4] A. G. Goodson and D. Grossman, "Strategies for early melanoma detection: approaches to the patient with nevi," Journal of the American Academy of Dermatology, vol. 60, no. 5, pp. 719-735, 2009.

[5] K. T. Flaherty, F. S. Hodi, and D. E. Fisher, "From genes to drugs: targeted strategies for melanoma," Nature Reviews. Cancer, vol. 12, no. 5, pp. 349-361, 2012.

[6] P. B. Chapman, A. Hauschild, C. Robert et al., "Improved survival with vemurafenib in melanoma with BRAF V600E mutation," The New England Journal of Medicine, vol. 364, no. 26, pp. 2507-2516, 2011.
[7] W. E. Damsky and M. Bosenberg, "Melanocytic nevi and melanoma: unraveling a complex relationship," Oncogene, vol. 36, no. 42, pp. 5771-5792, 2017.

[8] J. A. Curtin, J. Fridlyand, T. Kageshita et al., "Distinct sets of genetic alterations in melanoma," The New England Journal of Medicine, vol. 353, no. 20, pp. 2135-2147, 2005.

[9] A. H. Shain, I. Yeh, I. Kovalyshyn et al., "The genetic evolution of melanoma from precursor lesions," The New England Journal of Medicine, vol. 373, no. 20, pp. 19261936, 2015.

[10] W. H. Clark, D. E. Elder, D. Guerry, M. N. Epstein, M. H. Greene, and M. Van Horn, "A study of tumor progression: the precursor lesions of superficial spreading and nodular melanoma," Human Pathology, vol. 15, no. 12, pp. 1147-1165, 1984.

[11] W. M. Lin, S. Luo, A. Muzikansky et al., "Outcome of patients with de novo versus nevus-associated melanoma," Journal of the American Academy of Dermatology, vol. 72, no. 1, pp. 54-58, 2015.

[12] H. A. Haenssle, N. Mograby, A. Ngassa et al., "Association of patient risk factors and frequency of nevus-associated cutaneous melanomas," JAMA Dermatology, vol. 152, no. 3, pp. 291298, 2016.

[13] D. Shitara, G. Tell-Martí, C. Badenas et al., "Mutational status of naevus-associated melanomas," The British Journal of Dermatology, vol. 173, no. 3, pp. 671-680, 2015.

[14] S. Yang, T. Liu, H. Nan et al., "Comprehensive analysis of prognostic immune-related genes in the tumor microenvironment of cutaneous melanoma," Journal of Cellular Physiology, vol. 235, no. 2, pp. 1025-1035, 2020.

[15] J. Wu, T. Vallenius, K. Ovaska, J. Westermarck, T. P. Mäkelä, and S. Hautaniemi, "Integrated network analysis platform for protein-protein interactions," Nature Methods, vol. 6, no. 1, pp. 75-77, 2009.

[16] S. A. Bapat, A. Krishnan, A. D. Ghanate, A. P. Kusumbe, and R. S. Kalra, "Gene expression: protein interaction systems network modeling identifies transformation-associated molecules and pathways in ovarian cancer," Cancer Research, vol. 70, no. 12, pp. 4809-4819, 2010.

[17] R. Sharan, I. Ulitsky, and R. Shamir, "Network-based prediction of protein function," Molecular Systems Biology, vol. 3, no. 1, p. 88, 2007.

[18] R. Edgar, M. Domrachev, and A. E. Lash, "Gene Expression Omnibus: NCBI gene expression and hybridization array data repository," Nucleic Acids Research, vol. 30, no. 1, pp. 207-210, 2002.

[19] D. Talantov, A. Mazumder, J. X. Yu et al., "Novel genes associated with malignant melanoma but not benign melanocytic lesions," Clinical Cancer Research, vol. 11, no. 20, pp. 72347242, 2005.

[20] 1M. Scatolini, M. M. Grand, E. Grosso et al., "Altered molecular pathways in melanocytic lesions," International Journal of Cancer, vol. 126, no. 8, pp. 1869-1881, 2010.

[21] O. Kabbarah, C. Nogueira, B. Feng et al., "Integrative genome comparison of primary and metastatic melanomas," PLoS One, vol. 5, no. 5, article e10770, 2010.

[22] 1D. Huang, B. T. Sherman, Q. Tan et al., "The DAVID Gene Functional Classification Tool: a novel biological modulecentric algorithm to functionally analyze large gene lists," Genome Biology, vol. 8, no. 9, 2007. 
[23] M. Ashburner, C. A. Ball, J. A. Blake et al., "Gene ontology: tool for the unification of biology," Nature Genetics, vol. 25, no. 1, pp. 25-29, 2000.

[24] A. Franceschini, D. Szklarczyk, S. Frankild et al., "STRING v9.1: protein-protein interaction networks, with increased coverage and integration," Nucleic Acids Research, vol. 41, no. D1, pp. D808-D815, 2013.

[25] M. E. Smoot, K. Ono, J. Ruscheinski, P. L. Wang, and T. Ideker, "Cytoscape 2.8: new features for data integration and network visualization," Bioinformatics, vol. 27, no. 3, pp. 431-432, 2011.

[26] W. P. Bandettini, P. Kellman, C. Mancini et al., "MultiContrast Delayed Enhancement (MCODE) improves detection of subendocardial myocardial infarction by late gadolinium enhancement cardiovascular magnetic resonance: a clinical validation study," Journal of Cardiovascular Magnetic Resonance, vol. 14, no. 1, 2012.

[27] E. Cerami, J. Gao, U. Dogrusoz et al., "The cBio cancer genomics portal: an open platform for exploring multidimensional cancer genomics data," Cancer Discovery, vol. 2, no. 5, pp. 401404, 2012.

[28] G. Bindea, J. Galon, and B. Mlecnik, "CluePedia Cytoscape plugin: pathway insights using integrated experimental and in silico data," Bioinformatics, vol. 29, no. 5, pp. 661-663, 2013.

[29] H. H. Caicedo, D. A. Hashimoto, J. C. Caicedo, A. Pentland, and G. P. Pisano, "Overcoming barriers to early disease intervention,” Nature Biotechnology, vol. 38, no. 6, pp. 669-673, 2020.

[30] Z. Tang, C. Li, B. Kang, G. Gao, C. Li, and Z. Zhang, "GEPIA: a web server for cancer and normal gene expression profiling and interactive analyses," Nucleic Acids Research, vol. 45, no. W1, pp. W98-W102, 2017.

[31] W. H. Xu, S. N. Shi, Y. Xu et al., "Prognostic implications of aquaporin 9 expression in clear cell renal cell carcinoma," Journal of Translational Medicine, vol. 17, no. 1, 2019.

[32] A. Subramanian, P. Tamayo, V. K. Mootha et al., "Gene set enrichment analysis: a knowledge-based approach for interpreting genome-wide expression profiles," Proceedings of the National Academy of Sciences of the United States of America, vol. 102, no. 43, pp. 15545-15550, 2005.

[33] L. A. Taylor, C. O'Day, T. Dentchev et al., "p15 expression differentiates nevus from melanoma," The American Journal of Pathology, vol. 186, no. 12, pp. 3094-3099, 2016.

[34] D. J. Panka, M. B. Atkins, and J. W. Mier, "Targeting the mitogen-activated protein kinase pathway in the treatment of malignant melanoma," Clin Cancer Res, vol. 12, no. 7, pp. 2371s-2375s, 2006.

[35] A. Sharma, M. A. Tran, S. Liang et al., "Targeting mitogenactivated protein kinase/extracellular signal-regulated kinase kinase in the mutant (V600E) B-Raf signaling cascade effectively inhibits melanoma lung metastases," Cancer Research, vol. 66, no. 16, pp. 8200-8209, 2006.

[36] L. Danilova, H. Wang, J. Sunshine et al., "Association of PD1/PD-L axis expression with cytolytic activity, mutational load, and prognosis in melanoma and other solid tumors," Proceedings of the National Academy of Sciences of the United States of America, vol. 113, no. 48, pp. E7769-E7777, 2016.

[37] A. Najem, M. Krayem, F. Salès et al., "P53 and MITF/Bcl-2 identified as key pathways in the acquired resistance of NRAS-mutant melanoma to MEK inhibition," European Journal of Cancer, vol. 83, pp. 154-165, 2017.
[38] A. A. Adjei, "Blocking oncogenic Ras signaling for cancer therapy," Journal of the National Cancer Institute, vol. 93, no. 14, pp. 1062-1074, 2001.

[39] M. Liu, Y. du, H. Li et al., "Cyanidin-3-o-glucoside pharmacologically inhibits tumorigenesis via estrogen receptor $\beta$ in melanoma mice," Frontiers in Oncology, vol. 9, 2019.

[40] M. C. Brouard, J. H. Saurat, G. Ghanem, and G. Siegenthaler, "Urinary excretion of epidermal-type fatty acid-binding protein and S100A7 protein in patients with cutaneous melanoma," Melanoma Research, vol. 12, no. 6, pp. 627-631, 2002.

[41] R. Z. Liu, K. Graham, D. D. Glubrecht, D. R. Germain, J. R. Mackey, and R. Godbout, "Association of FABP5 expression with poor survival in triple-negative breast cancer: implication for retinoic acid therapy," The American Journal of Pathology, vol. 178, no. 3, pp. 997-1008, 2011.

[42] C. Wasinger, M. Künzl, C. Minichsdorfer, C. Höller, M. Zellner, and M. Hohenegger, "Autocrine secretion of 15dPGJ2 mediates simvastatin-induced apoptotic burst in human metastatic melanoma cells," British Journal of Pharmacology, vol. 171, no. 24, pp. 5708-5727, 2014.

[43] S. Mohapatra, R. S. Varma, L. M. S. Kumar et al., "Anti-skin ageing activity of napthoquinones from Arnebia nobilis Reichb.f.," Natural Product Research, vol. 30, no. 5, pp. 574$577,2016$.

[44] S. C. Chou, Y. Azuma, M. A. Varia, and J. A. Raleigh, "Evidence that involucrin, a marker for differentiation, is oxygen regulated in human squamous cell carcinomas," British Journal of Cancer, vol. 90, no. 3, pp. 728-735, 2004.

[45] H. Takahashi, Y. Hashimoto, A. Ishida-Yamamoto, and H. Iizuka, "Roxithromycin suppresses involucrin expression by modulation of activator protein- 1 and nuclear factor- $\kappa \mathrm{B}$ activities of keratinocytes," Journal of Dermatological Science, vol. 39, no. 3, pp. 175-182, 2005.

[46] Y. Ramot, E. Gáspár, A. Dendorfer, L. Langbein, and R. Paus, "The 'melanocyte-keratin' mystery revisited: neither normal human epidermal nor hair follicle melanocytes express keratin 16 or keratin 6 in situ," The British Journal of Dermatology, vol. 161, no. 4, pp. 933-938, 2009.

[47] K. M. Spaunhurst, A. M. Hogendorf, F. J. D. Smith et al., "Pachyonychia congenita patients with mutations in KRT6A have more extensive disease compared with patients who have mutations in KRT16," The British Journal of Dermatology, vol. 166, no. 4, pp. 875-878, 2012.

[48] L. M. López-Sánchez, B. Jurado-Gámez, N. Feu-Collado et al., "Exhaled breath condensate biomarkers for the early diagnosis of lung cancer using proteomics," American Journal of Physiology-Lung Cellular and Molecular Physiology, vol. 313, no. 4, pp. L664-L676, 2017.

[49] P. A. Rojas, M. May, G. R. Sequeira et al., "Progesterone receptor isoform ratio: a breast cancer prognostic and predictive factor for antiprogestin responsiveness," JNCI: Journal of the National Cancer Institute, vol. 109, no. 7, pp. 1-9, 2017.

[50] V. Giroux, J. Stephan, P. Chatterji et al., "Mouse intestinal _Krt15+_ crypt cells are radio-resistant and tumor initiating," Stem Cell Reports, vol. 10, no. 6, pp. 1947-1958, 2018.

[51] L. Ghali, S.-T. Wong, N. Tidman, A. Quinn, M. P. Philpott, and I. M. Leigh, "Epidermal and hair follicle progenitor cells express melanoma-associated chondroitin sulfate proteoglycan core protein," The Journal of Investigative Dermatology, vol. 122, no. 2, pp. 433-442, 2004. 
[52] L. X. Wang, Y. Li, and G. Z. Chen, "Network-based coexpression analysis for exploring the potential diagnostic biomarkers of metastatic melanoma," PLoS One, vol. 13, no. 1, article e0190447, 2018.

[53] R. Metri, A. Mohan, J. Nsengimana et al., "Identification of a gene signature for discriminating metastatic from primary melanoma using a molecular interaction network approach," Scientific Reports, vol. 7, no. 1, 2017.

[54] S. Zurac, M. Neagu, C. Constantin et al., "Variations in the expression of TIMP1, TIMP2 and TIMP3 in cutaneous melanoma with regression and their possible function as prognostic predictors," Oncology Letters, vol. 11, no. 5, pp. 3354-3360, 2016. 